\title{
The World in a Nutshell: Concise Range Queries
}

\author{
Ke Yi, Xiang Lian, Student Member, IEEE, Feifei Li, and Lei Chen, Member, IEEE
}

\begin{abstract}
With the advance of wireless communication technology, it is quite common for people to view maps or get related services from the handheld devices, such as mobile phones and PDAs. Range queries, as one of the most commonly used tools, are often posed by the users to retrieve needful information from a spatial database. However, due to the limits of communication bandwidth and hardware power of handheld devices, displaying all the results of a range query on a handheld device is neither communication efficient nor informative to the users. This is simply because that there are often too many results returned from a range query. In view of this problem, we present a novel idea that a concise representation of a specified size for the range query results, while incurring minimal information loss, shall be computed and returned to the user. Such a concise range query not only reduces communication costs, but also offers better usability to the users, providing an opportunity for interactive exploration.

The usefulness of the concise range queries is confirmed by comparing it with other possible alternatives, such as sampling and clustering. Unfortunately, we prove that finding the optimal representation with minimum information loss is an NP-hard problem. Therefore, we propose several effective and non-trivial algorithms to find a good approximate result. Extensive experiments on realworld data have demonstrated the effectiveness and efficiency of the proposed techniques.
\end{abstract}

Index Terms-Spatial databases, range queries, algorithms.

\section{INTRODUCTION}

Spatial databases have witnessed an increasing number of applications recently, partially due to the fast advance in the fields of mobile computing, embedded systems and the spread of the Internet. For example, it is quite common these days that people want to figure out the driving or walking directions from their handheld devices (mobile phones or PDAs). However, facing the huge amount of spatial data collected by various devices, such as sensors and satellites, and limited bandwidth and/or computing power of handheld devices, how to deliver light but usable results to the clients is a very interesting, and of course, challenging task.

Our work has the same motivation as several recent work on finding good representatives for large query answers, for example, representative skyline points in [1]. Furthermore, such requirements are not specific to spatial databases. General query processing for large relational databases and OLAP data warehouses has posed similar challenges. For example, approximate, scalable query processing has been a focal point in the recent work [2] where the goal is to provide light, usable representations of the query results early in the query processing stage, such that an interactive query process is possible. In fact, [2] argued to return concise representations of the final query results in every possible stage of a long-running query evaluation. However, the focus of [2] is on join queries in the relational database and

- Ke Yi, Xiang Lian, and Lei Chen are with the Department of Computer Science and Engineering, Hong Kong University of Science and Technology, Clear Water Bay, Hong Kong, China. Emails: \{yike,xlian,leichen\}@cse.ust.hk

- Feifei Li is with the Department of Computer Science, Florida State University, Tallahassee, FL, USA. Email: lifeifei@cs.fsu.edu the approximate representation is a random sample of the final query results. Soon we will see, the goal of this work is different and random sampling is not a good solution for our problem.

For our purpose, light refers to the fact that the representation of the query results must be small in size, and it is important for three reasons. First of all, the clientserver bandwidth is often limited. This is especially true for mobile computing and embedded systems, which prevents the communication of query results with a large size. Moreover, it is equally the same for applications with PCs over the Internet. In these scenarios, the response time is a very critical factor for attracting users to choose the service of a product among different alternatives, e.g., Google Map vs. Mapquest, since long response time may blemish the user experience. This is especially important when the query results have large scale. Secondly, clients' devices are often limited in both computational and memory resources. Large query results make it extremely difficult for clients to process, if not impossible. This is especially true for mobile computing and embedded systems. Thirdly, when the query result size is large, it puts a computational and I/O burden on the server. The database indexing community has devoted a lot of effort in designing various efficient index structures to speed up query processing, but the result size imposes an inherent lower bound on the query processing cost. If we return a small representation of the whole query results, there is also potential in reducing the processing cost on the server and getting around this lower bound. As we see, simply applying compression techniques only solves the first problem, but not the latter two.

Usability refers to the question of whether the user could derive meaningful knowledge from the query 
results. Note that more results do not necessarily imply better usability. On the contrary, too much information may do more harm than good, which is commonly known as the information overload problem. As a concrete example, suppose that a user issues a query to her GPS device to find restaurants in the downtown Boston area. Most readers having used a GPS device could quickly realize that the results returned in this case could be almost useless to the client for making a choice. The results (i.e., a large set of points) shown on the small screen of a handheld device may squeeze together and overlap. It is hard to differentiate them, let alone use this information! A properly sized representation of the results will actually improve usability. In addition, usability is often related to another component, namely, query interactiveness, that has become more and more important. Interactiveness refers to the capability of letting the user provide feedback to the server and refine the query results as he or she wishes. This is important as very often, the user would like to have a rough idea for a large region first, which provides valuable information to narrow down her query to specific regions. In the above example, it is much more meaningful to tell the user a few areas with high concentration of restaurants (possibly with additional attributes, such as Italian vs. American restaurants), so that she could further refine her query range.

\subsection{Problem definition}

Motivated by these observations this work introduces the concept of concise range queries, where concise collectively represents the light, usable, and interactive requirements laid out above. Formally, we represent a point set using a collection of bounding boxes and their associated counts as a concise representation of the point set.

Definition 1 Let $P$ be a set of $n$ points in $\mathbb{R}^{2}$. Let $\mathcal{P}=\left\{P_{1}, \ldots, P_{k}\right\}$ be a partitioning of the points in $P$ into $k$ pairwise disjoint subsets. For each subset $P_{i}$, let $R_{i}$ be the minimum axis-parallel bounding box of the points in $P_{i}$. Then the collection of pairs $\mathcal{R}=$ $\left\{\left(R_{1},\left|P_{1}\right|\right), \ldots,\left(R_{k},\left|P_{k}\right|\right)\right\}$ is said to be a concise representation of size $k$ for $P$, with $\mathcal{P}$ as its underlying partitioning.

We will only return $\mathcal{R}$ as a concise representation of a point set to the user, while the underlying partitioning $\mathcal{P}$ is only used by the DBMS for computing such an $\mathcal{R}$ internally. Note that the definition above can be easily extended to a set of objects of arbitrary shapes, rather than just points. In particular, in Section 4 we will apply the same notion on a set of rectangles. But for now we will focus on point sets. Clearly, for fixed dimensions the amount of bytes required to represent $\mathcal{R}$ is only determined by its size $k$ (as each box $R_{i}$ could be captured with its bottom left and top right corners).

There could be many possible concise representations for a given point set $P$ and a given $k$. Different representations could differ dramatically in terms of quality, as with $\mathcal{R}$, all points in a $P_{i}$ are replaced by just a bounding box $R_{i}$ and a count $\left|P_{i}\right|$. Intuitively, the smaller the $R_{i}$ 's are, the better. In addition, an $R_{i}$ that contains a large number of points shall be more important than one containing few points. Thus we use the following "information loss" as the quality measure of $\mathcal{R}$.

Definition 2 For a concise representation $\mathcal{R}=\left\{\left(R_{1},\left|P_{1}\right|\right), \ldots,\left(R_{k},\left|P_{k}\right|\right)\right\}$ of a point set $P$, its information loss is:

$$
L(\mathcal{R})=\sum_{i=1}^{k}\left(R_{i} \cdot \delta_{x}+R_{i} \cdot \delta_{y}\right)\left|P_{i}\right|,
$$

where $R_{i} . \delta_{x}$ and $R_{i} . \delta_{y}$ denote the $x$-span and $y$-span of $R_{i}$, respectively, and we term $R_{i} . \delta_{x}+R_{i} . \delta_{y}$ as the extent of $R_{i}$.

The rationale behind the above quality measure is the following. In the concise representation $\mathcal{R}$ of $P$, we only know that a point $p$ is inside $R_{i}$ for all $p \in P_{i}$. Therefore, the information loss as defined in (1) is the amount of "uncertainty" in both the $x$-coordinate and the $y$-coordinate of $p$, summed over all points $p$ in $P$.

A very relevant problem is the $k$-anonymity problem from the privacy preservation domain, which observed the problem from a completely different angle. In fact, both $k$-anonymity and the concise representation could be viewed as clustering problems with the same objective function (1). After obtaining the partitioning $\mathcal{P}$, both of them replace all points in each subset $P_{i}$ with its bounding box $R_{i}$. However, the key difference is that $k$-anonymity requires each cluster to contain at least $k$ points (in order to preserve privacy) but no constraint on the number of clusters, whereas in our case the number of clusters is $k$ while there is no constraint on cluster size. Extensive research on the $k$-anonymity [3], [4], [5] has demonstrated the effectiveness of using (1) as a measure of the amount of information loss by converting the point set $P$ into $\mathcal{R}$.

Now, with Definitions 1 and 2, we define concise range queries.

Definition 3 Given a large point set $P$ in $\mathbb{R}^{2}$, a concise range query $Q$ with budget $k$ asks for a concise representation $\mathcal{R}$ of size $k$ with the minimum information loss for the point set $P \cap Q$.

Therefore, the user can specify a $k$ according to the bandwidth and/or computing power of her mobile device, and retrieve the query results in a concise format meeting her constraints. On the other hand, we can also fix the information loss $L$ and seek for a concise representation with a minimum $k$ (the size of the output), which is a complement problem of Definition 3. Formally,

Definition 4 Given a large point set $P$ in $\mathbb{R}^{2}$, a complement concise range query $Q$ with budget $L$ asks for a concise 
representation $\mathcal{R}$ of a minimum size, i.e., minimum $k$, for the point set $P \cap Q$ with information loss $L(\mathcal{R}) \leq L$.

We will focus on Definition 3 and discuss in Section 5 on how to extend the proposed solutions to complement concise range queries.

\subsection{Summary of Contributions}

Therefore, the goal of a concise range query is to find a concise representation, with the user specified size, for all the points inside the query range. Ideally, one would like to have a concise representation of minimum information loss. We first give a dynamic programming algorithm that finds the optimal solution in one dimension in Section 3.1. Unfortunately, this optimization problem in two or more dimensions is NP-hard. In Section 3.2, we present a nontrivial reduction from PLANAR 3-SAT to the concise representation problem and prove its NPhardness. Nevertheless, in our applications, the optimal solution is often unnecessary while efficiency is typically important. Thus, in Section 3.3 we focus on designing efficient yet effective algorithms that find good (but not optimal) concise representations.

The definition as stated requires us to first find the point sets $P \cap Q$ before trying to solve the optimization problem, i.e., we first need to process $Q$ as if it were a standard "exact" range query. Since in our setting $Q$ is typically large, and we are not aiming at the optimal solution due to the NP-hardness, this process is often expensive and unnecessary. Noticing the fact that we can evaluate $L(\mathcal{R})$ by only looking at $\mathcal{R}$ itself without knowing $P \cap Q$, we can actually carry out the optimization process without computing $P \cap Q$ in its entirety. Then in Section 4, we explore how to speed up query processing by using an existing R-tree built on the data set $P$. We present an adaptive R-tree traversal algorithm that is much more efficient than answering the query exactly, and also produces high-quality concise representations of the query results.

We discuss some extensions of concise range queries in Section 5. In Section 6, we demonstrate the effectiveness and efficiency of the proposed techniques with extensive experiments on real data sets. A survey of related works appears in Section 7 before concluding the paper.

\section{Limitation OF OtHER ALTERNATIVES}

Clustering techniques: There is a natural connection between the concise range query problem and the many classic clustering problems, such as $k$-means, $k$ centers, and density based clustering. In fact, our problem could be interpreted as a new clustering problem if we return the underlying partitioning $\mathcal{P}$ instead of the concise representation $\mathcal{R}$. Similarly, for existing clustering problems one could return, instead of the actual clusters, only the "shapes" of the clusters and the numbers of points in the clusters. This will deliver a small representation of the data set as well. Unfortunately, as the primary goal of all the classic clustering problems is classification, the various clustering techniques do not constitute good solutions for our problem. In this section, we argue why this is the case and motivate the necessity of seeking new solutions tailored specifically for our problem.

Consider the example in Figure 1, which shows a typical distribution of interesting points (such as restaurants) near a city found in a spatial database. There are a large number of points in a relatively small downtown area. The suburbs have a moderate density while the points are sparsely located in the countryside. For illustration purposes we suppose the user has a budget $k=3$ on the concise representation.

The concise representation following our definition will partition this data set into three boxes as in Figure 1(a) (we omit the counts here). The downtown area is summarized with a small box with many points. The suburb is grouped by a larger box that overlaps with the first box (note that its associated count does not include those points contained in the first box) and all the outliers from the countryside are put into a very large box. One can verify that such a solution indeed minimizes the information loss (1). The intuition is that in order to minimize (1), we should partition the points in such a way that small boxes could have a lot of points while big boxes should contain as few as possible. If adding a new point to a cluster increases the size of its bounding box then we need to exercise extra care, as it is going to increase the "cost" of all the existing points in the cluster. In other words, the cost of each point in a cluster $C$ is determined by the "worst" points in $C$. It is this property that differentiates our problem with all other clustering problems, and actually makes our definition an ideal choice for obtaining a good concise representation of the point set.

The result of using the modified $k$-means approach is shown in Figure 1(b). Here we also use the bounding box as the "shape" of the clusters. (Note that using the (center, radius) pair would be even worse.) Recall that the objective function of $k$-means is the sum of distance (or distance squared) of each point to its closest center. Thus in this example, this function will be dominated by the downtown points, so all the 3 centers will be put in that area, and all the bounding boxes are large. This obviously is not a good representation of the point set: It is not too different from that of, say, a uniformly distributed data set.

One may argue that the result in Figure 1(b) is due to the presence of outliers. Indeed, there has been a lot of work on outlier detection, and noise-robust clustering [6]. However, even if we assume that the outliers can be perfectly removed and hence the bounding boxes can be reduced, it still does not solve the problem of putting all three centers in the downtown (Figure 1(c)). As a result, roughly $1 / 3$ of the downtown points are mixed together with the suburban points. Another potential problem is, what if some of the outliers are important? Although it is 


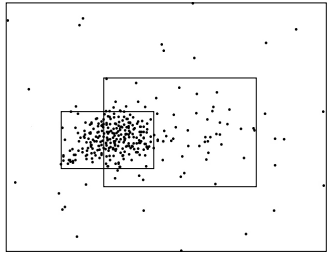

(a) our result.

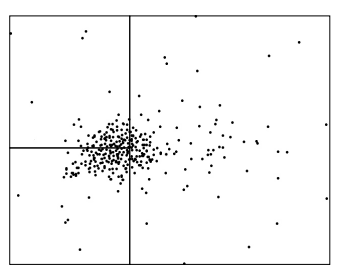

(b) $k$-means.

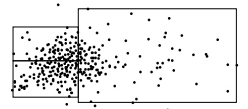

(c) liers.

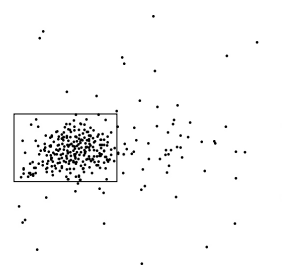

ing.

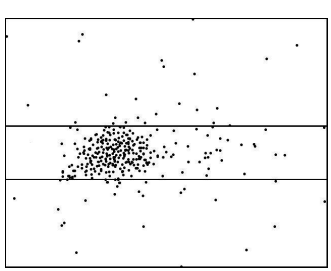

(e) MinSkew.

Fig. 1. Different alternatives for defining the concise representation, $k=3$.

not necessary to pinpoint their exact locations, the user might still want to know their existence and which region they are located in. Our representation (Figure 1(a)) with $k=3$ only tells the existence of these outliers. But as we increase $k$, these outliers will eventually be partitioned into a few bounding boxes, providing the user with more and more information about them.

Lastly, Figure 1(d) shows the result obtained by a density based clustering approach. A typical density based clustering, such as CLARANS [7], discovers the clusters by specifying a clustering distance $\epsilon$. After randomly selecting a starting point for a cluster, the cluster starts to grow by inserting neighbors whose distance to some current point in the cluster is less than $\epsilon$. This process stops when the cluster cannot grow any more. This technique, when applied to our setting, has two major problems. First, we may not find enough clusters for a given $k$ (assume that there is a support threshold on the minimum number of points in one cluster). In this example we will always have only one cluster. Secondly, the clusters are quite sensitive to the parameter $\epsilon$. Specifically, if we set $\epsilon$ small, then we will obtain only the downtown cluster (Figure 1(d)); if we set $\epsilon$ large, then we will obtain the cluster containing both the downtown and the suburb. Neither choice gives us a good representation of the point set.

Finally, we omit the result from $k$-centers. Since $k$ centers is trying to create clusters that minimize the maximum distance of any point to its cluster center, it is easy to see that this produces even worse results than $k$-means with respect to being good concise representations of the input data set. In addition, histogram [28], [29], [30] is also a popular tool for summarizing data in several buckets (partitions), however, the construction of histogram has a different goal from our problem. We will give a detailed discussion later in Section 7.

In summary, none of the clustering techniques work well for the concise range query problem since the primary goal of clustering is classification. To the contrary, as shown in Figure 1(a), our goal is to build the partitioning $\mathcal{P}$ that minimizes the information loss. Hence, we need to look for new algorithms and techniques for the concise range query problem, which consciously .

Histogram: Our work is also related to histogram construction [28], [29], [30]. Specifically, a histogram consists of several buckets, each of which stores the frequency of data points in it. The histogram has been widely used as a tool for selectivity estimation [28], [29]. Ioannidis and Poosala [28] studied the V-optimal histogram for 1-dimensional data, whereas Acharya et al. [29] investigated the histogram, namely MinSkew, in multidimensional space for spatial data. The construction of V-optimal/MinSkew histogram aims to find partitions to minimize the error of selectivity estimation (or called spatial-skew), which is defined as summing up the multiplication of frequency and the statistical variance of the spatial densities of all points grouped within that bucket. Thus, the goal of V-optimal/MinSkew histogram is different from ours (i.e. minimizing the information loss). Another major difference is that MinSkew does not allow overlapping buckets, whereas we do. Figure 1(e) illustrates an example of MinSkew, resulting in disjoint partitions of the data set. Note, however, that here the 3 obtained buckets only minimize the spatialskew, rather than the information loss (which is defined as summing up the multiplication of frequency and the extent of each bucket).

Random sampling: Random sampling is another tempting choice, but it is easy to see that it is inferior to our result in the sense that, in order to give the user a reasonable idea on the data set, a sufficient number of samples need to be drawn, especially for skewed data distributions. For example, using $k=3$ bounding boxes roughly corresponds to taking 6 random samples. With a high concentration of points in the downtown area, it is very likely that all 6 samples are drawn from there.

Indeed, random sampling is a very general solution that can be applied on any type of queries. In fact, the seminal work of [2] proposed to use a random sample as an approximate representation of the results of a join, and designed nontrivial algorithms to compute such a random sample at the early stages of the query execution process. The fundamental difference between their work and ours is that the results returned by a range query in a spatial database are strongly correlated by the underlying geometry. For instance, if two points $p$ and $q$ are returned, then all the points in the database that lie inside the bounding box of $p$ and $q$ must also be returned. Such a property does not exist in the query results of a join. Thus, it is difficult to devise more effective approximate representations for the results of joins than random sampling. On the other hand, due to the nice 
geometric and distributional properties exhibited by the range query results, it is possible to design much more effective means to represent them concisely. Our work is exactly trying to exploit these nice spatial properties, and design more effective and efficient techniques tailored for range queries.

\section{The Base Algorithms}

In this section, we focus on the problem of finding a concise representation for a point set $P$ with the minimum information loss. First in Section 3.1, we show that in one dimension, a simple dynamic programming algorithm finds the optimal solution in polynomial time. However, this problem becomes NP-hard in two dimensions as we show in the Section 3.2. Then we settle for efficient heuristic algorithms for the problem in Section 3.3 for two or higher dimensions.

\subsection{Optimal solution in one dimension}

We first give a dynamic programming algorithm for computing the optimal concise representation for a set of points $P$ lying on a line. Later in Section 3.3 we will extend it to higher dimensions, leading to an efficient heuristic.

Let $p_{1}, \ldots, p_{n}$ be the points of $P$ in sorted order. Let $\mathcal{P}_{i, j}$ represent the optimal partitioning underlying the best concise representation, i.e., with the minimum information loss, for the first $i$ points of size $j, i \geq j$. The optimal solution is simply the concise representation for $\mathcal{P}_{n, k}$, and $\mathcal{P}_{n, k}$ could be found using a dynamic programming approach. The key observation is that in one dimension, the optimal partitioning always contains segments that do not overlap, i.e., we should always create a group with consecutive points without any point from another group in-between. Formally, we have

Lemma $1 \mathcal{P}_{i, j}$ for $i \leq n, j \leq k$ and $i \geq j$ assigns $p_{1}, \ldots, p_{i}$ into $j$ non-overlapping groups and each group contains all consecutive points covered by its extent.

Proof: We prove by contradiction. Suppose this is not the case and $\mathcal{P}_{i, j}$ contains two groups $P_{1}$ and $P_{2}$ that overlap in their extents as illustrated in Figure 2. Let $P_{i} . x_{l}$ and $P_{i} \cdot x_{r}$ denote the leftmost and rightmost points in $P_{i}$. Without loss of generality we assume $P_{1} \cdot x_{l} \leq$ $P_{2} . x_{l}$. Since $P_{1}$ intersects $P_{2}$, we have $P_{2} . x_{l} \leq P_{1} \cdot x_{r}$. If we simply exchange the membership of $P_{1} \cdot x_{r}$ and $P_{2} . x_{l}$ to get $P_{1}^{\prime}$ and $P_{2}^{\prime}$, it is not hard to see that both groups' extents shrink and the numbers of points stay the same. This contradicts with the assumption that $\mathcal{P}_{i, j}$ is the optimal partitioning.

Thus, $\mathcal{P}_{i, j}$ is the partitioning with the smallest information loss from the following $i-j+1$ choices: $\quad\left(\mathcal{P}_{i-1, j-1},\left\{p_{i}\right\}\right), \quad\left(\mathcal{P}_{i-2, j-1},\left\{p_{i-1}, p_{i}\right\}\right)$, $\left.\ldots,\left(\mathcal{P}_{j-1, j-1},\left\{p_{j}, \ldots, p_{i}\right\}\right)\right\}$. Letting $L\left(\mathcal{P}_{i, j}\right)$ be the information loss of $\mathcal{P}_{i, j}$, the following dynamic programming formulation becomes immediate.

$$
L\left(\mathcal{P}_{i, j}\right)=\min _{1 \leq \ell \leq i-j+1}\left(L\left(\mathcal{P}_{i-\ell, j-1}\right)+\ell \cdot\left|p_{i}-p_{i-\ell+1}\right|\right),
$$

for $1 \leq i \leq n, 2 \leq j \leq k$ and $j \leq i$. The base case is $\mathcal{P}_{i, 1}=\left\{\left\{p_{1}, \ldots, p_{i}\right\}\right\}$ for $1 \leq i \leq n$. Since computing each $L\left(\mathcal{P}_{i, j}\right)$ takes $O(n)$ time, the total running time is of this algorithm $O\left(k n^{2}\right)$.

Theorem 1 In one dimension, the concise representation with the minimum information loss for a set of points $P$ can be found in $O\left(k n^{2}\right)$ time.

We notice that there is a 1-dimensional approach given in [3], which is similar to the problem mentioned in this subsection with the only difference on the condition for the dynamic programming.

\subsection{Hardness of the problem in 2D}

Not surprisingly, like many other clustering problems, for a point set $P$ in $\mathbb{R}^{2}$, the problem of finding a concise representation of size $k$ with minimum information loss is NP-hard. Also similar to other clustering problems in Euclidean space, the NP-hardness proof is quite involved. Below, we give a carefully designed construction that gives a reduction from PLANAR 3-SAT to the concise representation problem.

In the classical 3-SAT problem, there are $n$ Boolean variables $x_{1}, \ldots, x_{n}$ and $m$ clauses $C_{1}, \ldots, C_{m}$, where each clause is a disjunction of three variables or their negations. The problem is to decide if there exists an assignment of variables such that all clauses evaluate to true. Assume that we map each variable and each clause to a vertex of an undirected graph $G$. Then, two nodes $u$ and $v$ in graph $G$ are connected if and only if $u$ represents a clause that contains the variable (or its negation) represented by $v$. If $G$ is planar, the problem is known as PLANAR 3-SAT. It is an NP-complete problem [8]. The problem remains NP-complete even if each variable and its negation appear in at most 3 clauses. Note that in this case, any vertex in $G$ has degree at most 3.

Given an instance of PLANAR 3-SAT, we construct a point set $P$ as follows. First, since any vertex in $G$ has degree at most 3, we can find a planar embedding of $G$ such that all the vertices lie on grid points and all the edges follow non-intersecting grid paths. Such an embedding can be found in $O(n+m)$ time and the resulting embedding has area $O\left((n+m)^{2}\right)$ [9].

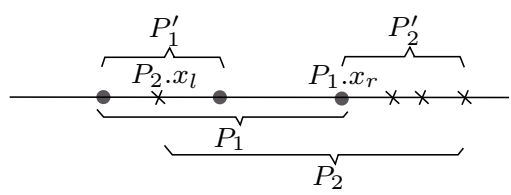

Fig. 2. Proof of Lemma 1. 


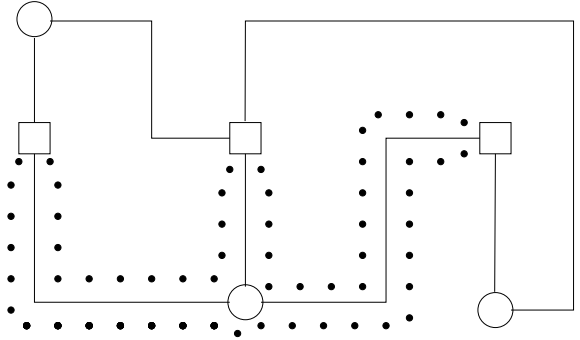

Fig. 3. The graph $G$ of the PLANAR 3-SAT instance, where a circle represents a variable and a square represents a clause. The figure shows the chain of points surrounding the 3 edges incident to one of the variables.

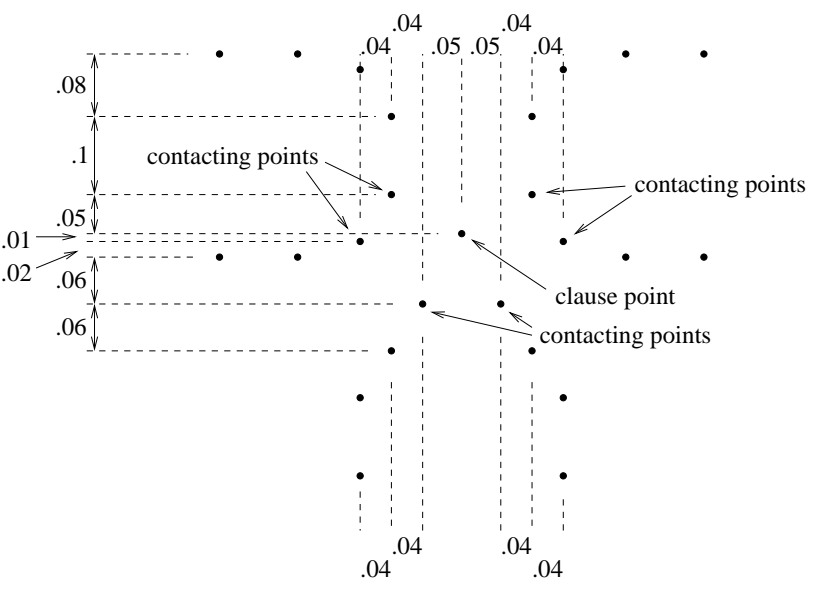

Fig. 4. Joining 3 chains at a clause.

For each variable $x_{i}$, we build a chain of an even number of points $\left(p_{i, 1}, \ldots, p_{i, 2 t_{i}}\right)$ surrounding the 3 incident edges of the variable vertex (Figure 3 ), such that the $\ell_{1}$ distance between any two consecutive points in the chain (including between $p_{i, 2 t_{i}}$ and $p_{i, 1}$ ) is 0.1. We also create a point in $P$ at each clause vertex $C_{j}$, where we join the 3 chains corresponding to the 3 variables in the clause using the configuration in Figure 4. Suppose $x_{i}$ (or $\bar{x}_{i}$ ) appears in $C_{j}$, and consider the two nearest $\ell_{1}$-neighbors in the chain of $x_{i}$. We refer to them as $C_{j}$ 's contacting points in the chain of $x_{i}$. Note that the $\ell_{1}$ distance from $C_{j}$ to them are both 0.14 . If $x_{i}$ appears in $C_{j}$, then we design the chain such that the two contacting points are $x_{i, 2 l-1}$ and $x_{i, 2 l}$ for some integer $l$; if $\bar{x}_{i}$ appears in $C_{j}$, then the two contacting points are $x_{i, 2 l}$ and $x_{i, 2 l+1}$ for some integer $l$. This is always doable by appropriately detouring the chain with additional points. This completes our construction of the point set $P$. It is obvious that it takes polynomial time. Finally, we set $k=\sum_{i} t_{i}$.

The basic intuition in our reduction is the following. We will partition each chain into $t_{i}$ pairs of points; this gives us $k$ subsets of $P$. There are two ways to partition the chain, namely $\left\{\left\{p_{i, 1}, p_{i, 2}\right\},\left\{p_{i, 3}, p_{i, 4}\right\}, \ldots,\left\{p_{i, 2 t_{i}-1}, p_{i, 2 t_{i}}\right\}\right\} \quad$ and $\left\{\left\{p_{i, 2 t_{i}}, p_{i, 1}\right\},\left\{p_{i, 2}, p_{i, 3}\right\}, \ldots,\left\{p_{i, 2 t_{i}-2}, p_{i, 2 t_{i}-1}\right\}\right\} . \quad$ Both of them have the same minimum information loss for the chain, and they will correspond to assigning "true" and "false" to the variable. Next we add each clause point $C_{j}$ into one of the pairs in one of its joining chains. By the way how we choose the contacting points, if $x_{i}$ appears in $C_{j}$ and $x_{i}=$ true, then we will be able to add $C_{j}$ into the pair of the two contacting points in $x_{i}$ 's chain, resulting in a bounding box with an extent of 0.19 . Similarly we can do the same if $\bar{x}_{i}$ appears in $C_{j}$ and $x_{i}=$ false. But if none of the variables satisfies the clause, $C_{j}$ will have to be added to a pair consisting of a contacting point and a non-contacting point, which will result in a bounding box with an extent $>0.19$. Thus we will be able to decide if the original PLANAR 3-SAT instance is satisfiable or not by looking at the information loss of the optimal concise representation of $P$. In the sequel, we formalize the idea by proving that the optimal partitioning of $P$ will exactly behave in the way we have prescribed above.

First, one can verify that the constructed point set $P$ has the following properties.

Property 1 The $\ell_{1}$ distance between any two consecutive points in any chain is 0.1 , while the distance between any other pair of points is $>0.1$.

Property 2 The bounding box for a clause point and the two contacting points from one of its joining chains has extent 0.19 . The bounding box for any other three points has extent $>0.19$.

Property 3 The extent of the bounding box is $\geq 0.24$ for any 4 points, $\geq 0.32$ for any 5 points, $\geq 0.36$ for any 6 points, and $\geq 0.38$ for any 7 points.

Let $\mathcal{R}_{\text {opt }}$ be the optimal concise representation for $P$ of size $k$. We first prove the following lemmas.

Lemma $2 L\left(\mathcal{R}_{o p t}\right) \geq 0.2 k+0.37 m$, and the lower bound can be attained only if each clause point is grouped together with the two contacting points from one of its joining chains, while each of the remaining points in $P$ is grouped together with one of its adjacent neighbors in its chain.

Proof: Please refer to Appendix A.

Lemma 3 The PLANAR 3-SAT instance has a satisfying assignment if and only if $L\left(\mathcal{R}_{\text {opt }}\right)=0.2 k+0.37 \mathrm{~m}$.

Proof: Please refer to Appendix B.

The following hardness result is an immediate consequence of Lemma 3.

Theorem 2 Given a point set $P \subset \mathbb{R}^{2}$ and an integer $k$, the problem of finding a concise representation $\mathcal{R}$ of size $k$ for $P$ with the minimum information loss is NP-hard. 


\subsection{Heuristics for two or more dimensions}

Given the hardness result, it is very unlikely that we can find an optimal concise representation $\mathcal{R}$ for a given point set $P$ in polynomial time in two or more dimensions. Thus in this subsection we try to design efficient heuristic algorithms that produces an $\mathcal{R}$ with low information loss, although not minimum. Since our problem is also a clustering problem, it is tempting to use some popular clustering heuristic, such as the wellknown $k$-means algorithm, for our problem as well. However, since the object function makes a big difference in different clustering problems, the heuristics designed for other clustering problems do not work for our case. The $k$-anonymity problem does share the same object function with us, but the clustering constraint there is that each cluster has at least $k$ points, while we require that the number of clusters is $k$. These subtle but crucial differences call for new heuristics to be tailored just for the concise representation problem.

Algorithm HGroup: Given the optimal algorithm in one dimension, a straightforward idea is to use a function $\mathbb{R}^{d} \rightarrow \mathbb{R}$ to map the points of $P$ from higher dimensions down to one dimension. Such an ordering function must somehow preserve the proximity relationships among the points. Many techniques exist for such a purpose (see [10] for a detailed review), among them the space-filling curves [11] have proved to be a popular choice. A space-filling curve traverses the space in a predetermined order. The most widely used space-filling curve is the Hilbert curve. The $h$-th order Hilbert curve has $2^{h d}$ cells in $d$ dimensions and visits all the cells in the manner shown in Figure 5. Each cell will be assigned a Hilbert value in sequence starting from 0 , and all points falling inside the cell will get the same Hilbert value. For example, the point shown in Figure 5 has a Hilbert value of 5 .

Our Hilbert-curve based algorithm, called HGroup, is shown in Algorithm 1. The basic idea is first compute the Hilbert value for each point in $P$, and sort all points by this value, mapping them to one dimension. Then, we simply group these points using our one-dimensional dynamic programming algorithm from Section 3.1. More precisely, equation (2) becomes

$L\left(\mathcal{P}_{i, j}\right)=\min _{1 \leq \ell \leq i-j+1}\left(L\left(\mathcal{P}_{i-\ell, j-1}\right)+\ell \cdot \operatorname{extent}\left(\left\{p_{i-\ell+1}, \ldots, p_{i}\right\}\right)\right.$

where the extent is calculated using points' Hilbert values in one dimension. Thus the running time of HGroup is still $O\left(k n^{2}\right)$, where $n$ is the size of $P$. Of course, the optimality of the algorithm will be lost, and the quality of the result will depend on how well the Hilbert curve preserves the neighborhood information among points in the original, higher-dimensional space [12]. It is not hard to imagine that the performance of HGroup will not be the best one can hope for, as important spatial information could be missed by the mapping of points from two dimension into one dimension. Hence, it is natural to design better algorithms that work in

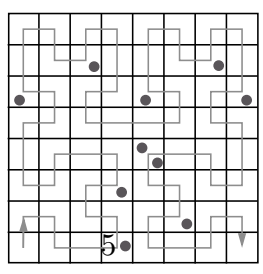

Fig. 5. The 3rd-order Hilbert curve in two dimensions.

two dimensions directly. On the other hand, HGroup is appealing by its simplicity and efficiency.

As a final note, the mapping from multidimensional data to 1-dimensional ones via Hilbert or iDistance [10] is also studied in [3]. Thus, we do not claim the HGroup approach is our major contribution.

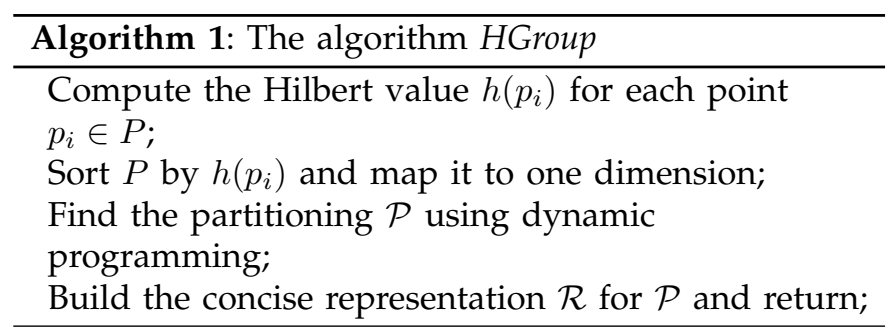

Algorithm IGroup: Below we present another, more direct algorithm in two or more dimensions. It is an iterative algorithm that finds the $k$ groups $P_{1}, \ldots, P_{k}$, one at a time. We call this algorithm IGroup. In each iteration, we start with a seed, randomly chosen from the remaining points, and greedily add points into the group one by one. In the $i$-th iteration, we first initialize the group $P_{i}$ to include only the seed. Let $U$ be the set of remaining points. Let $\rho(X)$ and $A(X)$ be the extent and area of the minimum bounding box of the set of points $X$, respectively. As we add points into $P_{i}$, we keep an estimated total information loss $\tilde{L}\left(P_{i}\right)$ for the current $P_{i}$, defined as

$$
\tilde{L}\left(P_{i}\right)=\rho\left(P_{i}\right)\left|P_{i}\right|+2 \sqrt{A(U) /(k-i)} \cdot|U| .
$$

Note that the first term in $\tilde{L}\left(P_{i}\right)$ is the information loss of $P_{i}$, while the second term is an estimate of the information loss on the remaining points $U$, assuming they are uniformly distributed in the bounding box of $U$.

When deciding which point $p \in U$ should be added to $P_{i}$, we first compute $\stackrel{L}{L}\left(P_{i} \cup\{p\}\right)$ for all $p \in U$, and choose the one that minimizes $\tilde{L}\left(P_{i} \cup\{p\}\right)$. If for all $p \in U, \tilde{L}\left(P_{i} \cup\{p\}\right) \geq \tilde{L}\left(P_{i}\right)$, then we stop adding points to $P_{i}$. The intuition is that, if we have $k-i$ bounding boxes left to group the points in $U$, then one partitioning that is always doable is to draw $k-i$ squares, each of dimensions (roughly) $\sqrt{A(U) /(k-i)} \times \sqrt{A(U) /(k-i)}$, to enclose all the points, which result in an information loss equal to the second term of $\tilde{L}\left(P_{i}\right)$. If $\tilde{L}\left(P_{i}\right)$ cannot be further reduced by adding more points, we should probably stop and start a new group. 
When we stop adding points and obtain a $P_{i}$, we record that together with its $\tilde{L}\left(P_{i}\right)$ achieved in the end, and call it a candidate. To improve quality, we carry out the same process with a number of different seeds, and choose the best candidate that has attained the lowest $\tilde{L}\left(P_{i}\right)$. Then we proceed to the next group $P_{i+1}$. Finally, for the last group $P_{k}$, we simply put all the remaining points into it. We give the details of the complete algorithm IGroup in Algorithm 2.

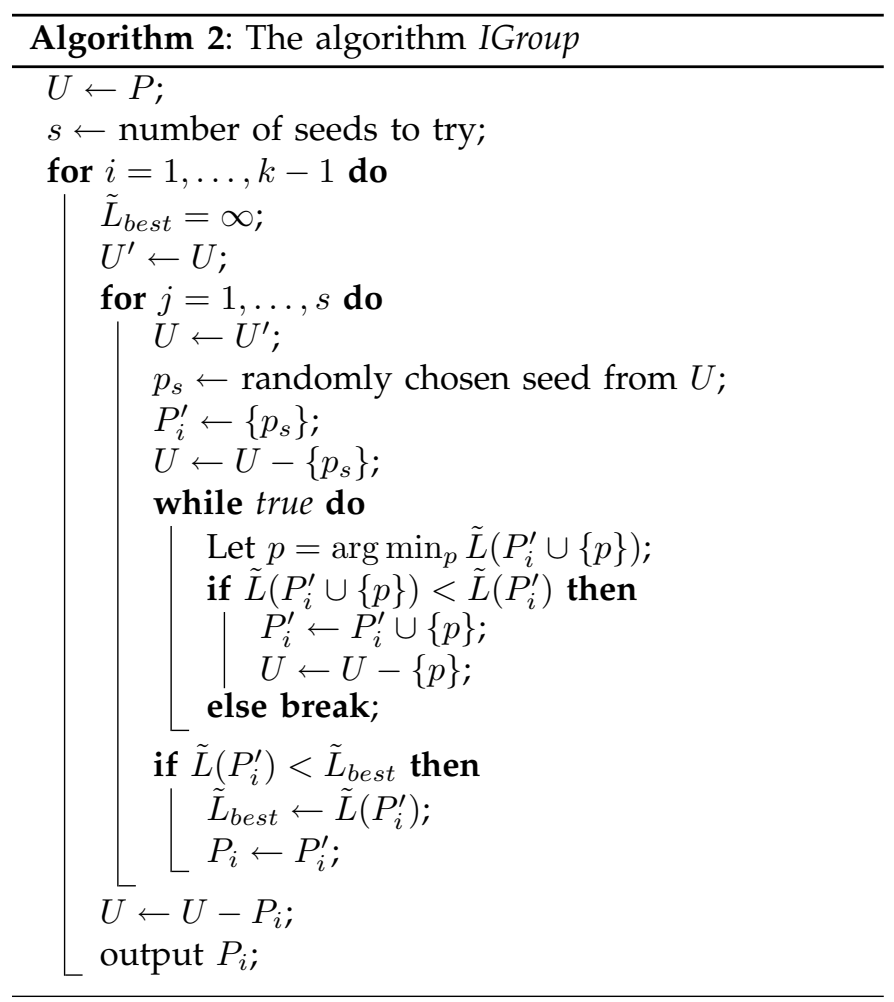

There are $k-1$ iterations in the algorithm. In each iteration, we check each of the $n$ points and choose the best one to add to the current group. In the worst-case, we could check all the points $O(n)$ times. Each iteration needs to be repeated for $s$ times with $s$ randomly chosen seeds. So the worst-case running time of IGroup is $O\left(s k n^{2}\right)$.

\section{Query Processing With R-trees}

In order to use the algorithms of Section 3.3 to answer a concise range query $Q$ with budget $k$ from the client, the database server would first need to evaluate the query as if it were a standard range query using some spatial index built on the point set $P$, typically an R-tree. After obtaining the complete query results $P \cap Q$, the server then partitions $P \cap Q$ into $k$ groups and returns the concise representation. However, as the main motivation to obtain a concise answer is exactly because $P \cap Q$ is too large, finding the entire $P \cap Q$ and running the algorithms of Section 3.3 are often too expensive for the database server. In this section, we present algorithms that process the concise range query without computing $P \cap Q$ in

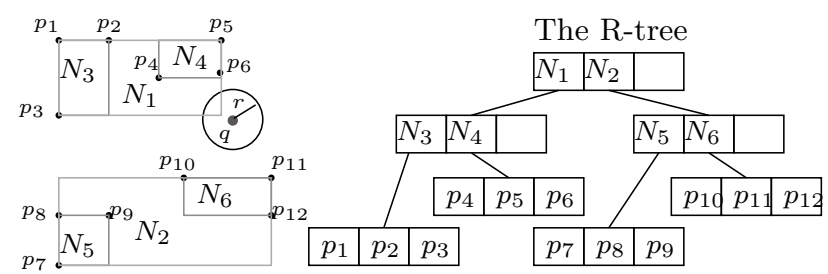

Fig. 6. The R-tree.

its entirety. The idea is to first find $k^{\prime}$ bounding boxes, for some $k^{\prime}>k$, that collectively contain all the points in $P \cap Q$ by using the existing spatial index structure on $P$. Each of these bounding boxes is also associated with the count of points inside. Then we run a weighted version of the algorithm in Section 3.3, grouping these $k^{\prime}$ bounding boxes into $k$ larger bounding boxes to form the concise representation $\mathcal{R}$. Typically $k^{\prime} \ll|P \cap Q|$, so we could expect significant savings in terms of I/O and CPU costs as compared with answering the query exactly. Therefore, adopting concise range queries instead of the traditional exact range queries not only solves the bandwidth and usability problems, but also leads to substantial efficiency improvements.

The algorithms presented in this section in general work with any space-partitioning index structure; for concreteness we will proceed with the R-tree, which is arguably the most widely used spatial index structure. The R-tree [13] and its variants ( $\mathrm{R}^{*}$-tree in particular [14]) all have the following structure. Suppose the disk block size is $B$. We first group $\leq B$ points in proximity area into a minimum bounding rectangle (MBR); these points will be stored at a leaf on the R-tree. These MBRs are then further grouped together level by level until there is only one left. Each node $u$ in the R-tree is associated with the MBR enclosing all the points stored below, denoted by $\operatorname{MBR}(u)$. Each internal node also stores the MBRs of all its children. An example of the R-tree is illustrated in Figure 6. Different R-tree variants only differ in the rules how the MBRs or points are grouped together.

The standard range query $Q$ can be processed using an R-tree as follows. We start from the root of the Rtree, and check the MBR of each of its children. Then we recursively visit any node $u$ whose MBR intersects or falls inside $Q$. When we reach a leaf, we simply return all the points stored there that are inside $Q$.

In this section, we in addition assume that each node $u$ in the R-tree also keeps $n_{u}$, the number of the points stored below its subtree. Such counts can be easily computed and maintained in the R-tree.

Basic ideas: In the following, for brevity we will also say a point is the MBR of itself. The basic idea of our R-tree based algorithms is to evaluate $Q$ in a way similar to a standard range query, but stop early in the tree so that we find $k^{\prime}$ MBRs to feed to the algorithm in Section 3.3, for some $\alpha \cdot k \leq k^{\prime} \ll|P \cap Q|$, where $\alpha>1$ is some constant. The observation is that, since the R-tree always tries to group close objects together with 
MBRs, these MBRs should be a good representation of the query results. However, on the other hand, since the primary goal of the R-tree is fast query processing, the MBRs do not constitute a good concise representation of the query result. For example, most R-tree variants try to minimize the overlap between MBRs, but as we have seen in Section 2, overlapping among the $R_{i}{ }^{\prime} \mathrm{s}$ is often necessary and beneficial for reducing the information loss. So we do not want to just use these MBRs as the $R_{i}$ 's in the concise representation $\mathcal{R}$. Instead we would like to find $k^{\prime} \geq \alpha k$ MBRs so that there is a chance for our grouping algorithm of Section 3.3 to further optimize the concise representation.

Algorithm R-BFS: The straightforward way to find $k^{\prime}$ such MBRs is to visit the part of the R-tree inside $Q$ in a BFS manner, until we reach a level where there are at least $\alpha k$ MBRs. We call this algorithm R-BFS. In particular, for any node $u$ whose MBR is complete inside $Q$, we directly return $\operatorname{MBR}(u)$ together with $n_{u}$. For any node $u$ whose MBR is partially inside $Q$, we return the intersection of the $\operatorname{MBR}(u)$ and $Q$, while the associated count is estimated as ${ }^{1}$

$$
n_{u} \cdot \frac{\operatorname{Area}(\operatorname{MBR}(u) \cap Q)}{\operatorname{Area}(Q)},
$$

assuming uniform distribution of the points in $\operatorname{MBR}(u)$.

Algorithm R-Adaptive: The above BFS traversal treats all nodes alike in the R-tree and will always stop at a single level. But intuitively, we should go deeper into regions that are more "interesting", i.e., regions deserving more user attention. These regions should get more budget from the $k$ bounding boxes to be returned to the user. Therefore, we would like a quantitative approach to measuring how "interesting" a node in the $\mathrm{R}$-tree is, and a corresponding traversal algorithm that visits the R-tree adaptively.

In the algorithm $R$-Adaptive, we start from the root of the R-tree with an initial budget of $\kappa=\alpha k$, and traverse the tree top-down recursively. Suppose we are at a node $u$ with budget $\kappa$, and $u$ has $b$ children $u_{1}, \ldots, u_{b}$ whose MBRs are either completely or partially inside $Q$. Let the counts associated with them be $n_{1}, \ldots, n_{b}$. Specifically, if $\operatorname{MBR}\left(u_{i}\right)$ is completely inside $Q$, we set $n_{i}=n_{u_{i}}$; if it is partially inside, we compute $n_{i}$ proportionally as in (4).

If $\kappa \leq b$, then we call the base algorithms of Section 3.3 to group them into $k$ larger MBRs, and return them. Otherwise, we allocate the budget $\kappa$ into $\kappa_{1}, \ldots, \kappa_{b}$, and assign $\kappa_{i}$ to $u_{i}$. For any $\kappa_{i}=1$, we directly return $\operatorname{MBR}\left(u_{i}\right)$ with $n_{i}$. If $\kappa_{i} \geq 2$, we recursively visit $u_{i}$ with budget $\kappa_{i}$.

It now remains to specify how we allocate the budget into $\kappa_{1}, \ldots, \kappa_{b}$. Intuitively, if $n_{i}$ is larger, then $\kappa_{i}$ should also be larger; on the other hand, if $\operatorname{MBR}\left(u_{i}\right)$ is larger, $\kappa_{i}$ should be larger too, since if two MBRs contain the same number of points, then we should allocate

1. Strictly speaking, when using the estimate (4), we will only return an approximate count for $\left|P_{i}\right|$ in the concise representation. But such an approximation is usually tolerable. more budget to the larger one in order to minimize the total information loss. So the allocation of budget should depend on a delicate balance between both $n_{i}$ and the $\operatorname{MBR}\left(u_{i}\right)$. Below we derive such an allocation policy assuming that all the points inside each $\operatorname{MBR}\left(u_{i}\right)$ are uniformly distributed and each $\operatorname{MBR}\left(u_{i}\right)$ is fat, i.e., having a bounded aspect ratio.

Let the area of $\operatorname{MBR}\left(u_{i}\right) \cap Q$ be $A_{i}$. If $\operatorname{MBR}\left(u_{i}\right)$ is fat, then when the $n_{i}$ points inside are grouped into $\kappa_{i}$ bounding boxes, each bounding box has extent roughly $2 \sqrt{A_{i} / \kappa_{i}}$ and contains $n_{i} / \kappa_{i}$ points. So the information loss for $u_{i}$ is $2 \sqrt{A_{i} / \kappa_{i}} \cdot n_{i} / \kappa_{i} \cdot \kappa_{i}=2 n_{i} \sqrt{A_{i} / \kappa_{i}}$. The total loss is thus

$$
L=2 n_{1} \sqrt{A_{1} / \kappa_{1}}+\cdots+2 n_{b} \sqrt{A_{b} / \kappa_{b}} .
$$

We now minimize $L$ under the constraint $\kappa_{1}+\cdots+\kappa_{b}=$ $\kappa$ but allowing the $\kappa_{i}$ 's to be real numbers.

By the Cauchy-Schwartz inequality, we have

$$
\begin{aligned}
L & =2 \frac{\left(\sum_{i} n_{i} \sqrt{A_{i} / \kappa_{i}}\right)\left(\sum_{i} \frac{\sqrt{\kappa_{i}}}{n_{i}^{1 / 3} A_{i}^{1 / 6}}\right)}{\sum_{i} \frac{\sqrt{\kappa_{i}}}{n_{i}^{1 / 3} A_{i}^{1 / 6}}} \\
& \geq 2 \frac{\left(\sum_{i} n_{i}^{1 / 3} A_{i}^{1 / 6}\right)^{2}}{\sum_{i} \frac{\sqrt{\kappa_{i}}}{n_{i}^{1 / 3} A_{i}^{1 / 6}}}
\end{aligned}
$$

while the denominator is

$$
\begin{aligned}
\sum_{i} \frac{\sqrt{\kappa_{i}}}{n_{i}^{1 / 3} A_{i}^{1 / 6}} & \leq \sqrt{\left(\sum_{i} \kappa_{i}\right)\left(\sum_{i} \frac{1}{n_{i}^{2 / 3} A_{i}^{1 / 3}}\right)} \\
& =\sqrt{\kappa\left(\sum_{i} \frac{1}{n_{i}^{2 / 3} A_{i}^{1 / 3}}\right) .}
\end{aligned}
$$

Both inequalities (5) and (6) become equality when $\kappa_{i} \propto n_{i}^{2 / 3} A_{i}^{1 / 3}$, for $i=1, \ldots, b$. Therefore, we set

$$
\kappa_{i}=\left\lceil\frac{n_{i}^{2 / 3} A_{i}^{1 / 3}}{\sum_{j} n_{j}^{2 / 3} A_{j}^{1 / 3}} \kappa\right\rceil .
$$

We use a ceiling in (7) since each $\kappa_{i}$ needs to be an integer, and the R-tree algorithm is allowed to return more MBRs than the budget. The final size $k$ in the concise representation will be enforced by the algorithm of Section 3.3. The procedure of the recursive call visit $(u, \kappa)$ is outlined in Algorithm 3. The process starts by calling visit (root of the R-tree, $\alpha k$ ).

The weighted versions of the base algorithms: Our R-tree based algorithms generate a number of MBRs, associated with counts, and pass them to the base algorithms of Section 3.3. As described those algorithms can only process a point set. But it is not difficult to adapt both HGroup and IGroup to handle a set of MBRs associated with counts (weights).

First, we simply take the centroids of each MBR to form the points in $P$ and each point has a weight that is equal to the count of the corresponding MBR. Then, for 


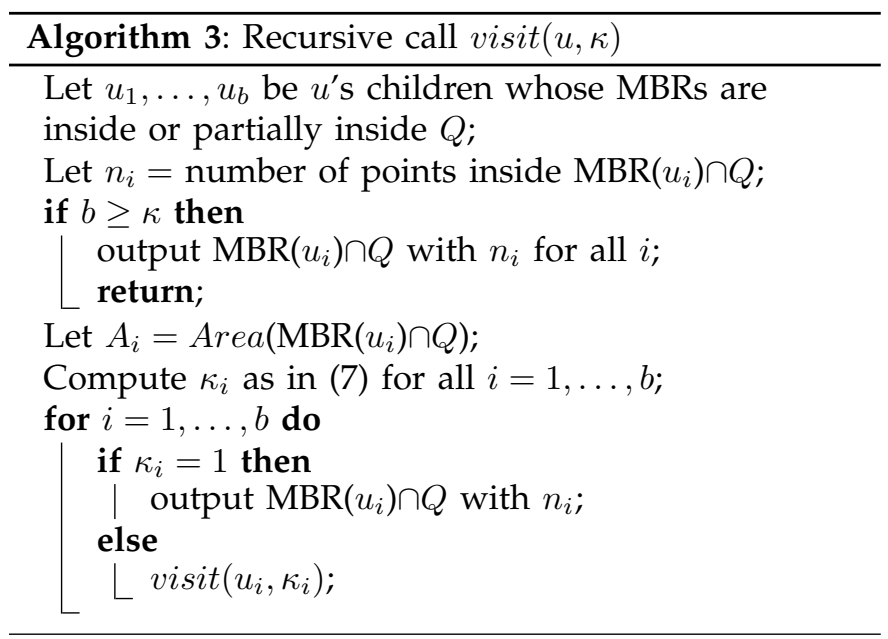

the dynamic programming algorithm (which is used in algorithm HGroup), we change equation (2) to

$$
\begin{aligned}
L\left(\mathcal{P}_{i, j}\right)= & \min _{\ell}\left(L\left(\mathcal{P}_{i-\ell, j-1}\right)+\operatorname{extent}\left(\left\{p_{i-\ell+1}, \ldots, p_{i}\right\}\right)\right. \\
& \left.\cdot \sum_{r=i-\ell+1}^{i} w\left(p_{r}\right)\right),
\end{aligned}
$$

where $w(p)$ denotes the weight of $p$.

For the algorithm IGroup, we simply update (3) as

$$
\tilde{L}\left(P_{i}\right)=\rho\left(P_{i}\right) \cdot \sum_{p \in P_{i}} w(p)+2 \sqrt{A(U) /(k-i)} \cdot \sum_{p \in U} w(p) .
$$

Discussions on the shape of query region: $U p$ to now, we always assume that the query region $Q$ is an axis-parallel rectangle. In the case where the query region $Q$ has other shapes (e.g. rotated rectangle, circle, or polygon), we can still apply our proposed approaches, $R$-BFS and R-Adaptive, with minor modifications. Specifically, for $R-B F S$ approach, the estimated count associated with node (partially intersecting with $Q$ ) in (4) has to consider the intersecting area between $\operatorname{MBR}(u)$ and $Q$ of any shape, which can be computed either by geometric position between the two, or by other estimation techniques such as histograms or sampling. Similarly, for $R$-Adaptive, we also need to consider the intersection between $\operatorname{MBR}(u)$ and $Q$ of any shape. We can obtain the area of the resulting intersection region via techniques mentioned above, and estimate the budget assigned to the MBR node (as given by (7)).

Discussions on other spatial data types: We now discuss how to extend our solutions of answering concise range queries on data points to that on other spatial data types such as lines or rectangles. In particular, although our proposed approaches are originally designed for answering concise queries over data points, they are still applicable to spatial data with other shapes. In fact, in the literature of spatial databases [14], spatial objects are often simplified by rectangles (which bound spatial objects with arbitrary shapes). Our extension of concise range queries on lines or rectangles can be as follows. First, we bound lines/rectangles with minimum bounding rectangles (MBRs). Then, we index the resulting MBRs in an R-tree structure by using the standard "insert" operator. For any specified concise range $Q$, we can conduct the concise range query on such MBRs via $\mathrm{R}$-tree in the same way as that over data points.

\section{EXTENSIONS}

Supporting attributes: As we motivated in Section 1, it is often useful if we can associate multiple counts with each bounding box, each of which represents the number of points with a particular attribute value, for example Italian, Chinese, and American restaurants. Such counts can be easily supported. We can augment each node of the R-tree with the number of objects that are stored below for each attribute value. Then, after we have computed the concise representation $\mathcal{R}$, we can easily also compute the number of points in each $P_{i}$ for each attribute value.

Complement concise range queries: As we mentioned in Section 1, the user may also be interested in asking a complement concise range query, i.e., she specifies a maximum allowed information loss $L$ and then the goal is to compute a concise representation of the query results with minimum size that has an information loss no more than $L$.

Since by definition, the larger $k$ is, the smaller the information loss is. Thus, we can conduct a binary search on $k$ to find the minimum $k$ that satisfies the user's requirement. For each $k$, we apply our algorithm in Section 3 and 4 to find a concise representation $\mathcal{R}$ and compare with $L$. Thus, after a logarithmic number of steps, we can find a good $\mathcal{R}$ that meets the user's requirement. Note that, however, since our heuristic solutions cannot guarantee to find the minimum information loss for a given $k$, this binary search cannot guarantee to find the minimum $k$ for the complement concise query problem, either. This is unsurprising, since the complement concise range query problem can be also shown to be NP-hard following our proof for the primary problem.

Progressive refinement: Another useful yet simple extension of our algorithms is to support progressive refinement of the concise representation. In this case the user with a slow network connection does not specify a $k$ beforehand. Instead the database server produces a series of concise representations of the query results with geometrically increasing sizes, e.g., $k=10,20,40,80, \ldots$. As time goes on, better and better representations are streamed to the user.

\section{EXPERIMENTS}

Experimental setup: We have implemented our two base algorithms, HGroup and IGroup, as well as the two R-tree traversal algorithms R-BFS and R-Adaptive. Specifically, we used the $R^{*}$-tree [14] to index all the points in the data set. The IGroup algorithms first traverse the Rtree to produce a number of MBRs and feed them into the base algorithms, so we have in total two combinations: $R$-BFS+IGroup, $R$-BFS+HGroup, $R$-Adaptive+IGroup, and $R$-Adaptive+HGroup. The straw-men we compare 


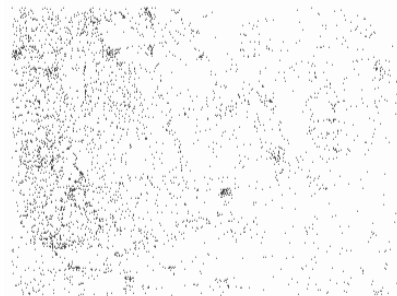

(a) All query results

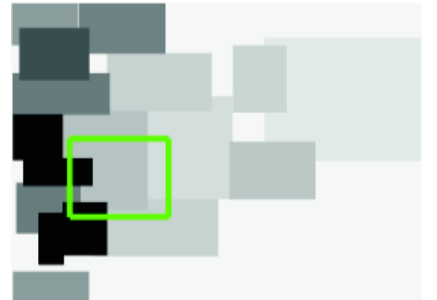

(b) Concise representation of (a)

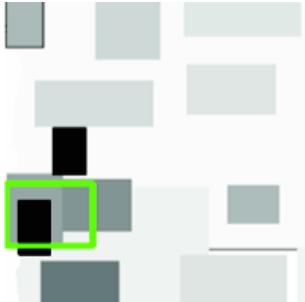
(b)

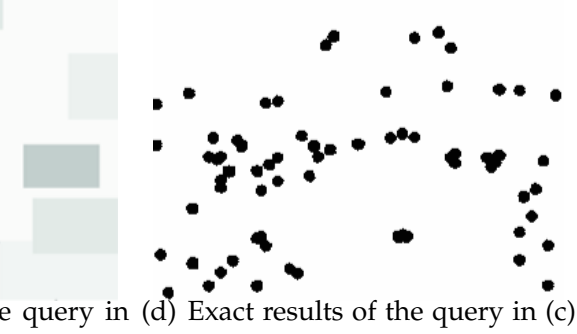

(c)
Fig. 7. Visualization of results.

against are $k$-means clustering algorithm, and MinSkew histogram [29]. In particular, for $k$-means and MinSkew, we first obtain all the data points in the query region via R-tree, and then conduct either of the two methods to obtain clusters/partitions. All the algorithms were implemented in $\mathrm{C}++$, and the experiments were conducted on a machine with a $4 \mathrm{GHz}$ CPU and $1 \mathrm{G}$ main memory. The page size of the R-tree is set to $4 \mathrm{~KB}$.

Data sets: We tested the query performance of our approaches over three real data sets, road networks from North America (NA), California (CA), and City of San Joaquin County (TG), and one synthetic data set, Skew. Specifically, for real data sets, NA and CA are from digital chart of the world server, whereas TG is from [31]. CA also contains a large number of points of interest (e.g., restaurants, resorts). These data sets are available online ${ }^{2}$. Points from the three real data sets have been normalized to lie within the unit square. The sizes for CA, NA, and TG are 125, 818, 175, 813, and 18263 , respectively. For the synthetic data set, Skew, we generate 100,000 data points within the unit square, where the coordinate on each dimension follows Zipf distribution (with skewness 0.8).

Note that, we evaluate the query performance on 2D real/synthetic point data sets mentioned above, since these spatial data follow typical data distributions in spatial databases. Moreover, the concise range query on these 2D spatial data has practical applications such as electronic maps like Google map or Mapquest, as mentioned in Section 1, where concise range answers have to be displayed on the small screens of handheld devices (e.g. PDA or mobile phone). Thus, we focus on evaluating 2D data sets in this section (though our proposed approaches can still hold for data sets with dimensionality greater than 2, as mentioned in Section 3.3).

Visualization of results and interactive exploration: We first did some test queries, and see if the concise representation indeed gives the user some intuitive high-level ideas about the query results. Figure 7(a) shows all the points that are returned by a range query of size $0.1 \times 0.1$ on the CA data set. There are a total of 4147 points in the full result, which corresponds to roughly $33 \mathrm{~K}$ bytes of data (assuming each coordinate and each count take 4 bytes). Figure 7(b) shows the

2. http://www.cs.fsu.edu/ lifeifei/SpatialDataset.htm concise representation of the results produced by our $R$ Adaptive+IGroup algorithm, where we used $k=20$ and $\alpha=8$. When plotting Figure 7(b), the gray scale is set to be proportional to the density of each bounding box (count/area). From the visualization results, we can see that even with $k$ as small as 20, which corresponds to merely 400 bytes (suppose the user's bandwidth budget is 400 bytes), the concise result is already a pretty close approximation of the exact result. Compared with the exact results, the concise representation has a 80 -fold reduction in size.

Starting from here, the user can interactively narrow her query down to areas of her interests, such as highdensity areas or areas with medium-density but closer to locations of interest, until the query range contains a small number of results, at which point all the exact results are returned. For example, Figure 7(c) shows the concise representation of the zoomed-in query, the green box, on Figure 7(b), while Figure 7(d) shows the exact results of the zoomed-in query, the green box, on Figure 7(c). Since the exact query results in Figure 7(d) can be represented with less than 400 bytes, all the points inside the query are returned exactly.

Note that, the resulting concise representations can be overlapping with each other. However, this is usually not a problem for the user perception, as long as we render the higher-density rectangles in front of the lowerdensity ones, such as in Fig. 7.

Experimental results with different approaches: We now compare six approaches $R$-BFS+IGroup, $R-$ Adaptive+IGroup, R-BFS+HGroup, R-Adaptive+HGroup, $k$ means, and MinSkew [29]. Figure 8 illustrates the query performance of the six approaches on CA data set, in terms of the number of $\mathrm{I} / \mathrm{O}$ cost (number of page accesses in the R-tree traversal), CPU time, and the average information loss per point in the concise representations returned. Note that, here the CPU time does not include the I/O cost. We fixed the query size at $0.1 \times 0.1, \alpha=8$, and $k=60$, and set the number of seeds in IGroup as $s=10$.

From Figure 8(a), the I/O costs of R-Adaptive+IGroup and $R$-Adaptive+HGroup are the lowest among six approaches, followed by R-BFS+IGroup and $R$ BFS+HGroup, and $k$-means and MinSkew are highest. This is because $k$-means and MinSkew have to retrieve all the data points (falling into the query region) in the 


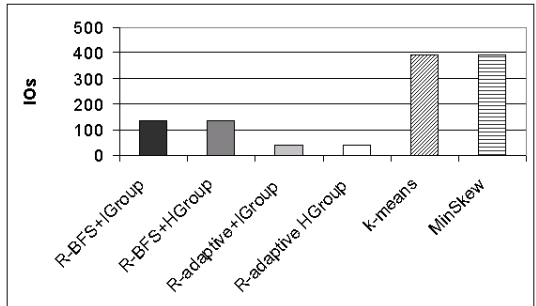

(a) $\mathrm{I} / \mathrm{O} \operatorname{cost}$

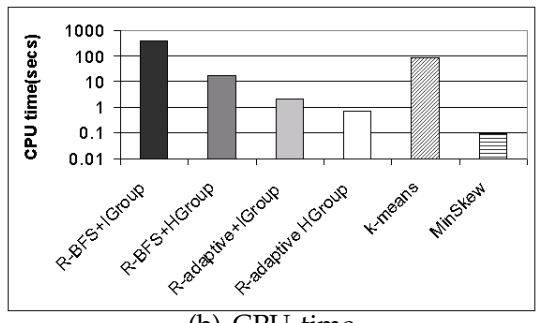

(b) CPU time

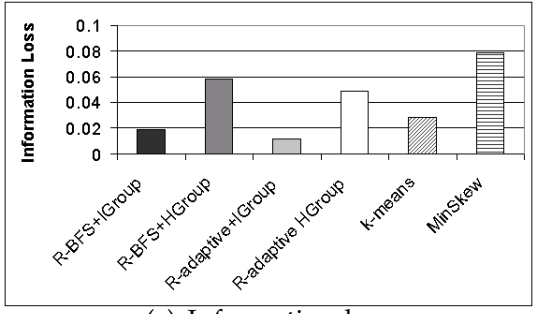

(c) Information loss

Fig. 8. Experimental results with different approaches on the CA data set.

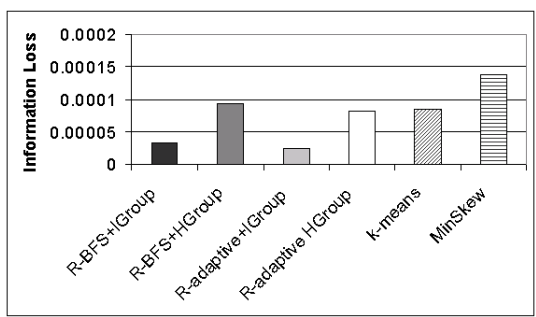

(a) NA

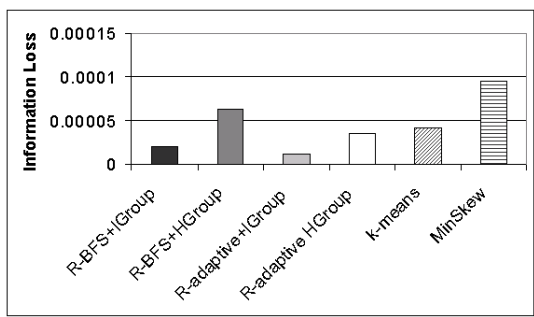

(b) TG

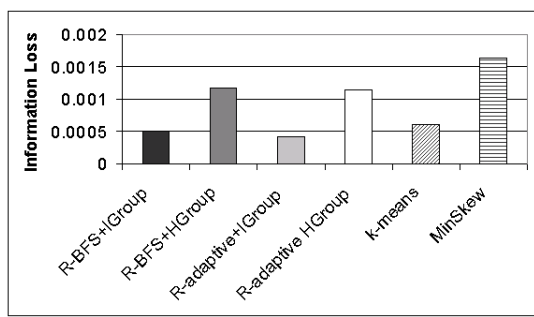

(c) Skew

Fig. 9. Information loss with different approaches on NA, TG, and Skew data sets.

leaf nodes, which incurs the largest number of page accesses.

In Figure 8(b), the CPU time of MinSkew is the lowest (about 0.1 second), due to the low cost of constructing the histogram. However, we can see from Figure 8(c) that MinSkew has the highest (worst) information loss among all approaches. The $k$-means algorithm incurs high CPU time (about 84 seconds) due to the data retrieval from the R-tree index and the clustering. Thus, $k$-means is not efficient in terms of CPU time (also true for the I/O cost), though the information loss in Figure 8(c) is larger than that of $R$-adaptive IGroup and $R$ BFS IGroup. From the experimental results, the HGroupbased approaches have good response time compared with the IGroup-based ones, however, with a bit high information loss. Thus, there is a trade-off between the query efficiency and information loss here. In practice, if small response time is strictly required by applications, we can use the HGroup-based approach to obtain fast answers; otherwise, the IGroup-based one can be used.

Figures 9(a), 9(b), and 9(c) show the average information loss per point on NA, TG, and Skew data sets, respectively, which has similar trend to $C A$ data set in Figure 8(c). The only difference is that, for NA and TG data sets, $k$-means method has even worse information loss than R-Adaptive+HGroup. The I/O cost and CPU time on these 3 data sets are similar to CA, and thus omitted due to space limit. From the discussions above, we can see that $k$-means incurs low query efficiency, and is worse than IGroup in terms of information loss; MinSkew returns answers fast, however, with high information loss (since MinSkew was not designed to minimize the information loss). Thus, they are not suitable for efficiently answering concise queries in applications like Google map on handheld devices. In the rest of this section, due to space limit, we only report the results on one real data set, CA, and one synthetic data set, Skew.
The trends for the other two real data sets, NA and TG, are similar and thus omitted.

Experimental results with varying $k$ : Next, we started to look into the performance of these six algorithms. In the first set of experiments, we fixed the query size at $0.1 \times 0.1, \alpha=8$, and varied $k$ from 20 to 100. Figures 10(a)-10(c) present experimental results on synthetic Skew data set, comparing the six approaches, whereas Figure 11 shows the same set of experiments on the real CA data set. Similar to previous results, for the Skew data set, the $k$-means and MinSkew incur higher I/O cost than the other four algorithms in Figure 10(a). Moreover, as shown in Figure 10(b), the CPU time of $k$ means is greater than that of $R$-adaptive IGroup, $R$-adaptive HGroup, and R-BFS HGroup. Although the CPU time of MinSkew is comparable to the R-adaptive HGroup, from Figure 10(c), MinSkew has the worst information loss among all the six approaches. Note that, the information loss of MinSkew in Figure 10(c) decreases slowly for larger $k$ values, compared with our approaches. This is because the MinSkew is designed for minimizing spatial-skew (rather than information loss). Thus, the MinSkew algorithm keeps on dividing buckets with the maximum decreases of spatial-skew (instead of maximum decreases of information loss). As a result, some large buckets with small variances (close to uniform), however, with high information losses, would not be divided by MinSkew (which is exactly the goal of the histogram construction); on the other hand, those buckets after splitting can achieve lower information loss, however, they only contribute a small portion of total information loss. As a consequence, when the number of buckets (i.e. $k$ ) becomes larger, the decreasing trend of information loss in MinSkew is small, compared with other approaches, as confirmed in Figure 10(c).

In the subsequent experiments, for the sake of clearly illustrating the trend of our approaches, we will 


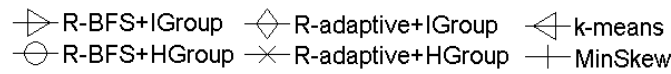

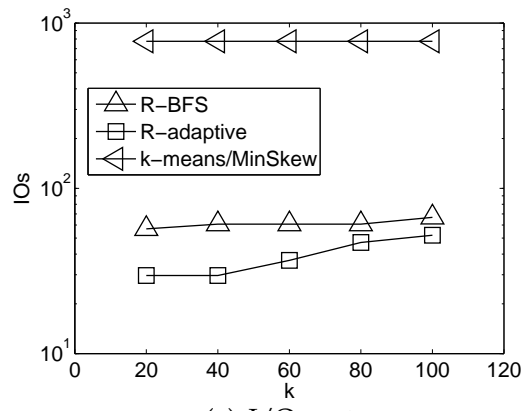

(a) $\mathrm{I} / \mathrm{O} \operatorname{cost}$

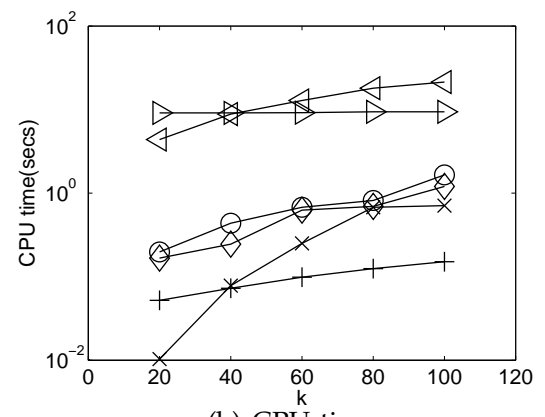

(b) CPU time

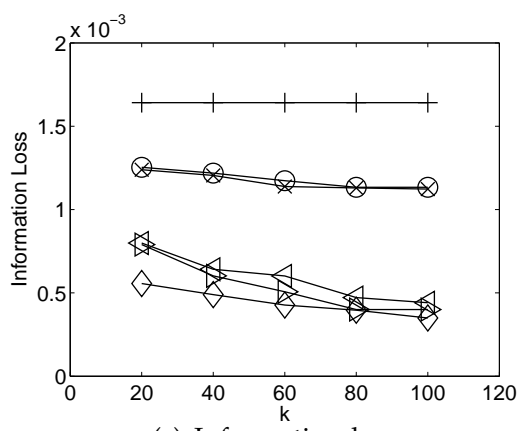

(c) Information loss

Fig. 10. Experimental results with varying $k$ on the Skew data set.

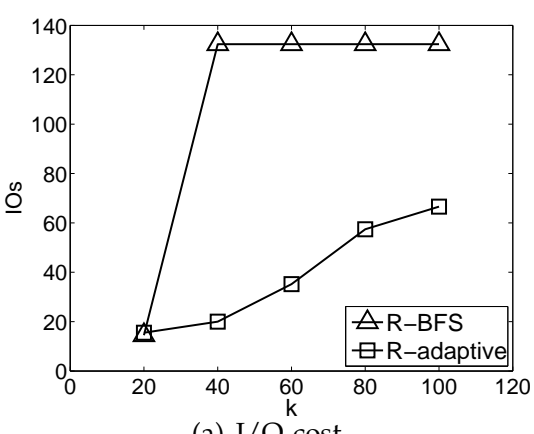

(a) $\mathrm{I} / \mathrm{O}$ cost

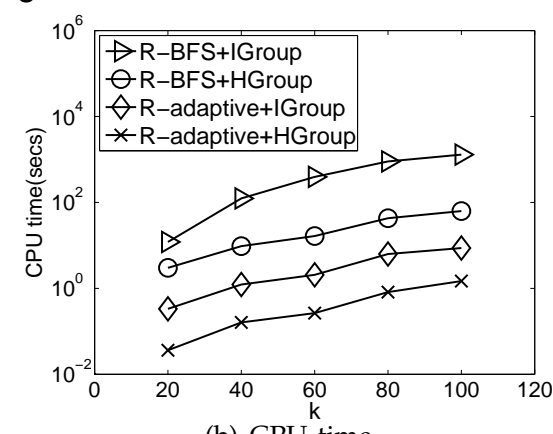

(b) CPU time

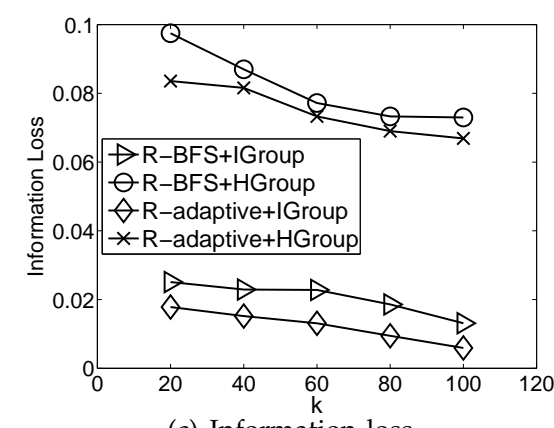

(c) Information loss

Fig. 11. Experimental results with varying $k$ on the CA data set.

only report the results with our four algorithms $R$ BFS+IGroup, R-Adaptive+IGroup, $R$-BFS+HGroup, and RAdaptive+HGroup. We next investigate the trend of our approaches for different $k$ values on CA data set in Figure 11. Specifically, Figure 11(a) shows the I/O cost of the four algorithms. Since the base algorithms do not incur any I/O, we only show the I/O costs of the two $\mathrm{R}$-tree algorithms. We can see that the number of page accesses of $R$-BFS increases quickly as $k$ gets larger. This is because it visits the R-tree indistinguishably. Once a certain level of the R-tree has less than $\alpha k$ MBRs in the query range, it visits all the nodes one level down. Thus the number of nodes accessed increases very quickly due to the large fan-out of the R-tree.

Figure 11(b) shows the CPU time of the four algorithms. First, we observe that the two variants using $R$ $B F S$ run much slower than the two R-Adaptive variants. This is expected, since the number of MBRs returned by $R$-BFS is much larger, and the running time is dominated by the grouping algorithms, which have complexities $O\left(k n^{2}\right)$ for HGroup and $O\left(s k n^{2}\right)$ for IGroup, respectively, where $n$ is the number of MBRs to be grouped. Comparing the two grouping algorithms, we observe that HGroup is faster than IGroup, which generally agrees with the analysis.

Figure 11(c) shows the average information loss per point in the concise representations returned, where we observe the following. First, the two IGroup based variants produce much better results than the two HGroup based variants. The explanation is possibly that, since the Hilbert curve imposes a linear order on the twodimensional data set, some important geometric properties are lost. While Hilbert curve based clustering algorithms [12] generally work well, our problem is fundamentally different from traditional clustering problems (as discussed in Section 2). Thus, simply extending the one-dimensional dynamic programming algorithm to two dimensions by Hilbert curves does not work as well as IGroup, which is directly designed for two dimensions. Secondly, coupled with the same grouping algorithm, $R$ Adaptive works much better than R-BFS. This means that visiting the $R$-tree more selectively does not only save the traversal cost, but also leads to better quality of the results. In addition, we observe that larger $k$ 's improve the quality for all the algorithms. This is intuitive, since as the size of the concise representation increases, it becomes closer and closer to the exact results. For NA and TG data sets, the query performance is similar and omitted due to space limit.

Experimental results with varying $\alpha$ : In the second set of experiments, we fixed the query size at $0.1 \times 0.1$, $k=60$, and varied $\alpha$ from 2 to 32. In Figure 12 we plot the I/O cost, CPU time, and information loss for the four algorithms on the CA data set. The trends on the I/O cost and CPU time in this set of experiments are similar to those in the experiments with varying $k$. This is not surprising, since both the I/O cost and CPU time depend only on the product $\alpha \cdot k$. The larger this 


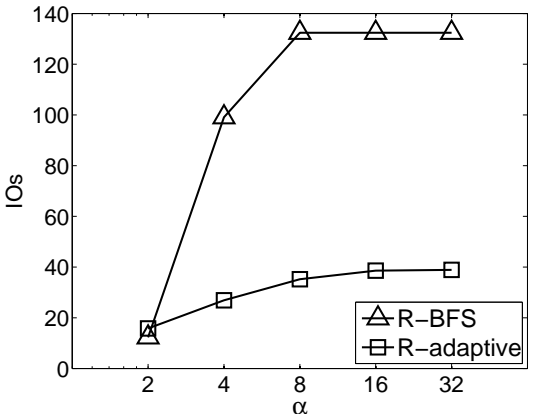

(a) $\mathrm{I} / \mathrm{O}$ cost

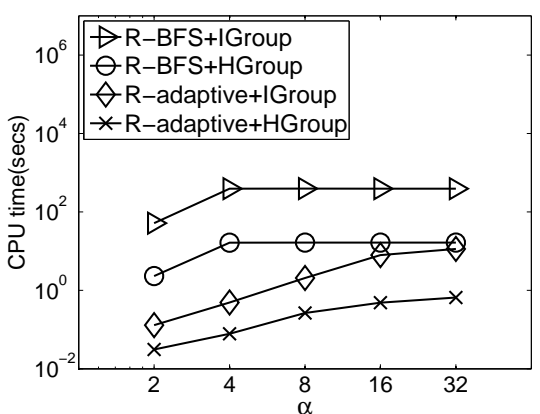

(b) CPU time

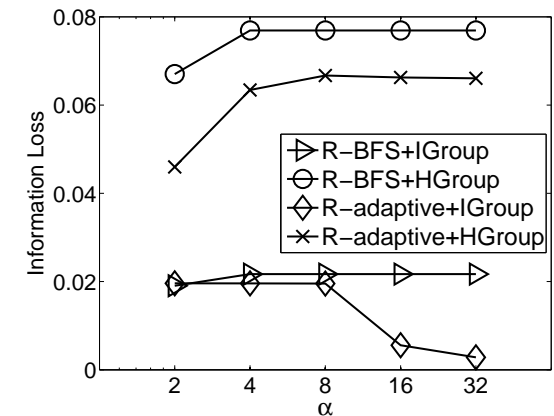

(c) Information loss

Fig. 12. Experimental results with varying $\alpha$ on the CA data set.

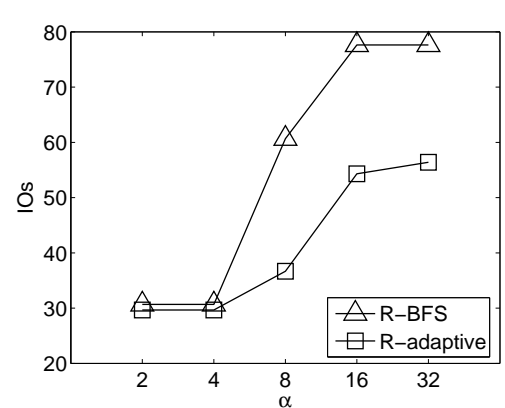

(a) $\mathrm{I} / \mathrm{O} \cos \mathrm{t}$

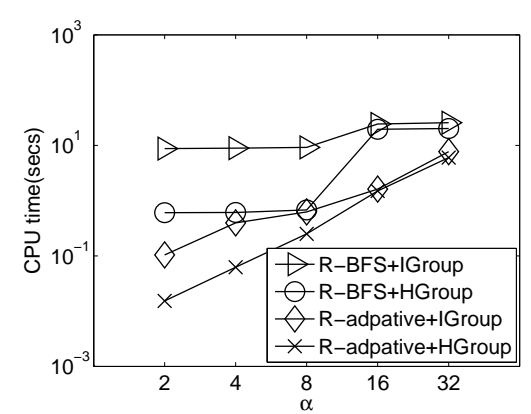

(b) CPU time

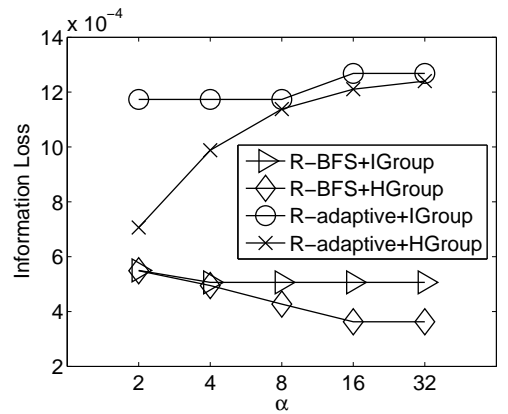

(c) Information loss

Fig. 13. Experimental results with varying $\alpha$ on the Skew data set.

product is, the more costly the algorithms are. Thus, the combination of $R$-Adaptive+IGroup is still the best in all algorithms in terms of information loss and its I/O cost.

For R-Adaptive+IGroup, we do see that larger $\alpha$ 's lead to better results since the R-tree traversal can return more candidate MBRs for IGroup to choose to form the partitions (Figure 12(c)). But we do not find the same trend with the other algorithms, which could mean that although $R$-BFS returns more candidate MBRs, they are of low quality. HGroup does not work well even with more freedom in grouping these MBRs, as long as the result size constraint, $k$, is fixed.

Furthermore, Figures 13(a)-13(c) illustrate the query performance on synthetic Skew data set with the same settings. The experimental results are similar to that of CA. We do not show the similar results on NA and TG data sets here due to space limit.

Experimental results with varying query size: In the last set of experiments, we fixed $k=60, \alpha=8$, and varied the query size from $0.05 \times 0.05$ to $0.2 \times 0.2$. The results on I/O cost, CPU time, and information loss on the CA data set are shown in Figure 14. First, similar to the previous two sets of experiments, the I/O cost of the algorithms increase as the query range gets larger. But this time the increase rate of $R$-BFS is slower than that when $\alpha \cdot k$ increases. This can be explained by the fact that, when $\alpha \cdot k$ increases, whenever a certain level of the R-tree does not contain enough MBRs, R-BFS simply goes one level down, increasing the number of MBRs returned exponentially. When $\alpha \cdot k$ is fixed but the query range gets larger, this increase is more "gradual": R-BFS will not go deep into the R-tree but rather go wide. So the number of page accesses increases more smoothly in this case. Nevertheless, the I/O cost of R-BFS is still much larger than that of $R$-Adaptive. In terms of CPU time, the trend is similar, i.e., all the algorithms take longer to run as the query gets larger. This is because of the high query selectivity, which requires more cost to retrieve and process data. Therefore, in practice, if small response time is strictly required (or the query region is large), we can use the HGroup-based approach to obtain fast answers; otherwise, the IGroup-based one can be used. Moreover, the information loss also gets larger for larger queries, which is not surprising. But across all queries, $R$ Adaptive+IGroup consistently beats all other alternatives.

Figures 15(a)-15(c) show the similar results on synthetic Skew data set under the same settings. For NA and TG data sets, the trends are similar and omitted due to space limit.

Finally, we also report the performance of our proposed four approaches with spatial objects of rectangular shape rather than data points. In particular, we first treat points in Skew data set as centers of objects, and then generate their (half) extents with a random value within $[0,1]$. The results are reported in Figure 16 in terms of I/O cost, CPU time, and information loss. We can see that the trend of our approaches on objects of rectangular shape is similar to that of point data sets.

\section{Related Work}

The motivation of this work is very similar to the recent work of Jermaine et al. [2]. The focus of [2] is 


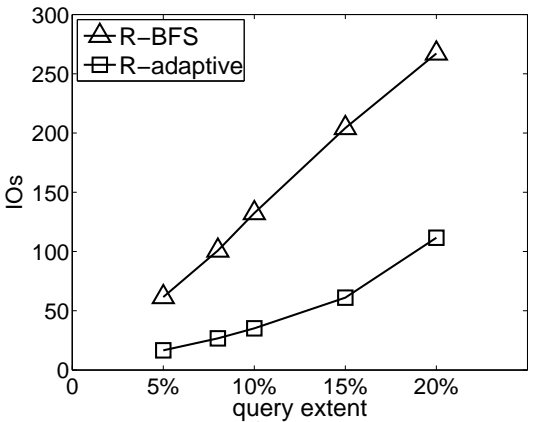

(a) $\mathrm{I} / \mathrm{O}$ cost

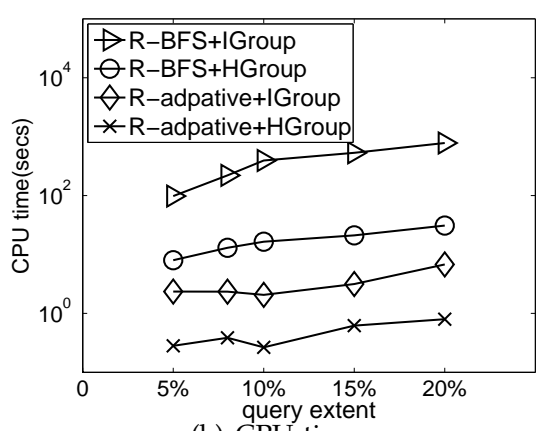

(b) CPU time

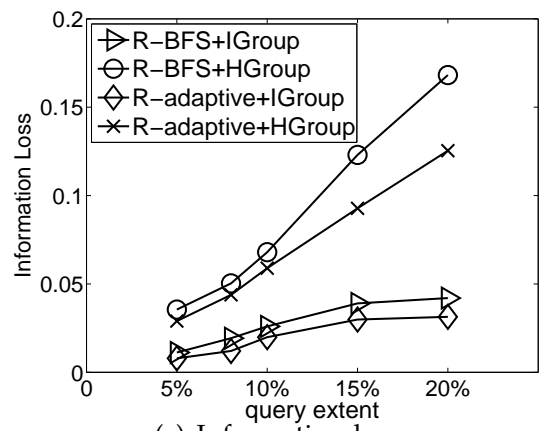

(c) Information loss

Fig. 14. Experimental results with varying query range on the CA data set.

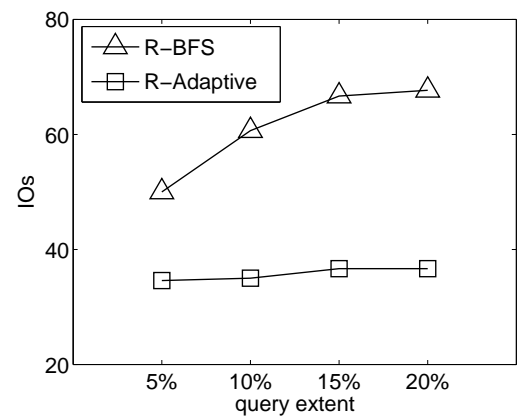

(a) I/O cost

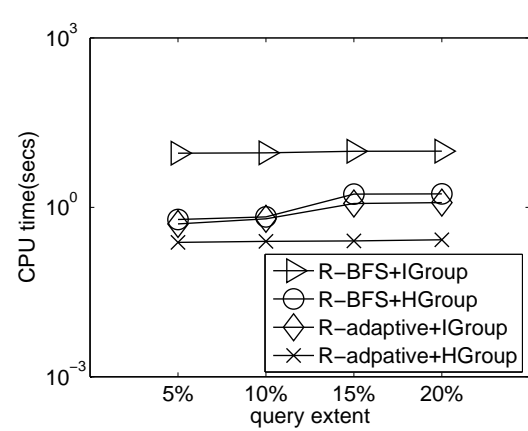

(b) CPU time

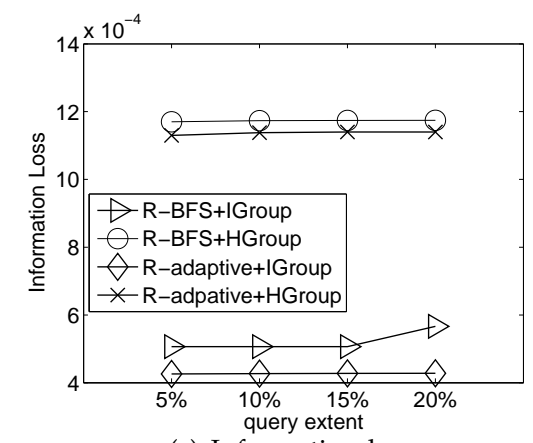

(c) Information loss

Fig. 15. Experimental results with varying query range on the Skew data set.

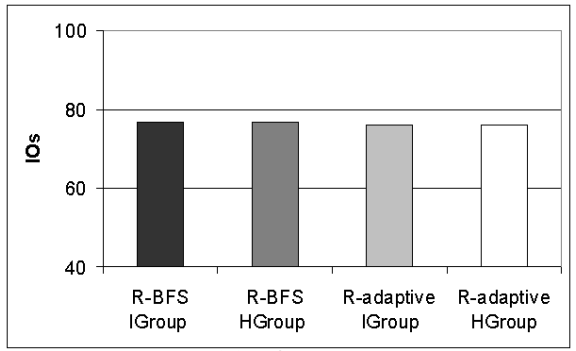

(a) $\mathrm{I} / \mathrm{O}$ cost

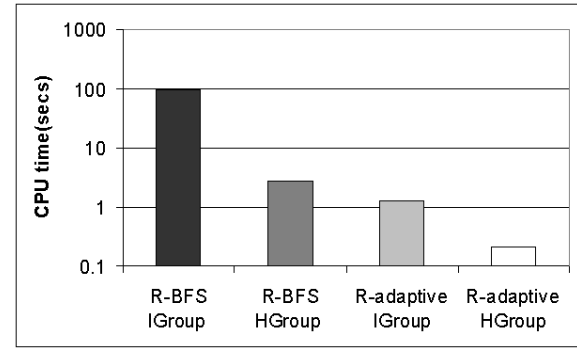

(b) CPU time

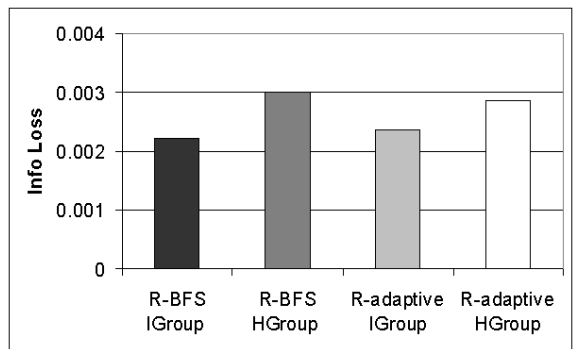

(c) Information loss

Fig. 16. Experimental results on Skew data set with objects of rectangular shape.

to produce approximate results for long-running join queries in a relational database engine at early stages of the query execution process. The "approximation" defined there is a random sample of the final results. As we elaborated in Section 2, due to the nice geometric properties of range queries in spatial databases, it is important to design more effective and efficient methods than random sampling. The goal of this work is thus to derive such a concise representation for range queries with the minimal amount of information loss. We also make use of multi-dimensional indexing structures to speed up the query answering process. Hence, although the motivations are similar, our ideas and techniques are fundamentally different from those in [2]. With similar arguments, our work also bears the same motivation as finding the representative skyline points [1], however, we focus on range queries rather than dominance points.

Section 2 has pointed out the close relationship be- tween the concise representation problem and classic clustering problems. I/O-efficient clustering algorithms have been studied in [15], [16]. In particular, $k$-medoids ( $k$-means with the constraint that the cluster center must be a point from the input data set) and $k$-centers have been extended to work for disk-based data sets using R-trees [17]. The basic idea is to explore the R-tree (in a top-down fashion) to the first level that provides enough nodes for clustering. Then some main memory heuristics are applied to these nodes. Of course, additional information, such as the means of all points within the subtree of one node and the total number of points in the same subtree, must be stored in R-tree nodes to enable this process. Our work focuses on a completely different definition of clustering, as Section 2 has illustrated the limitations of using either $k$-means or $k$-centers for our problem. Furthermore, instead of simply retrieving one level of the R-tree that provides enough nodes for deriv- 
ing $k$ partitions, we have designed an adaptive strategy to explore R-tree nodes at different levels.

Density based clustering approaches, such as CLARANS [7] and DBSCAN [18], are another popular clustering definition. Classic density-based clustering and other variants have been extended to work with disk-based data sets as well [15]. Several works have studied density-based clustering in the context of spatial databases and extended to road networks [19] and moving objects [20]. Similarly as above, Section 2 has examined why it is inappropriate to use as the concise representation. Nevertheless, an interesting open problem for our work is to study the concise range queries on road-networks [19] and for moving objects [20].

In addition, $\mathrm{Xu}$ et al. [5] studied the utility-based anonymization, which proposes heuristic local recoding methods for the privacy preserving, considering the utility of attributes. Although the proposed framework for the greedy top-down method is somewhat similar to IGroup, the fundamental problem (i.e. $k$-anonymity) is different from ours. As mentioned in Section 1.1, the main difference of our work from $k$-anonymity [3], [4], [5] is that, $k$-anonymity requires each cluster to contain at least $k$ points (for the sake of privacy preserving), rather than the number of clusters.

Our work is also related to the data summarization techniques such as histogram [28], [29], [30]. Specifically, Ioannidis and Poosala [28] studied the V-optimal histogram for 1-dimensional data, whereas Acharya et al. [29] investigated the histogram, namely MinSkew, in multidimensional space for spatial data. Note that, Section 3.1 discusses how to obtain optimal concise representations on 1-dimensional data, whose goal is however different from that of V-optimal histogram. That is, the goal of our problem is to minimize the information loss, which is defined as summing up the multiplication of frequency and the extent (i.e. the sum of spans of all dimensions) of each partition, rather than the estimation error. Furthermore, Jagadish et al. [30] worked on a dual problem of constructing a histogram, minimizing the space under the constraint of an acceptable error bound (for selectivity estimation). In contrast, our problem does not have the requirement of minimizing the space.

Finally, sampling-based techniques could be applied to derive concise representations for the results of range queries. Section 2 has argued that random sampling requires much larger sizes to be representative and they are also ineffective in capturing the outliers. The key observation is that sampling is a very general method that works for almost any type of data. Spatial data has special geometric properties that one should exploit. Other sampling techniques, such as the density biased sampling [21] and many others, have similar limitations in the context of range queries.

The basic idea of representing range queries in a concise format has been published as a short paper in ICDE'09 [22]. However, the only technical contents in
[22] are the basic one-dimensional dynamic programming algorithm and the HGroup algorithm. The NPhardness result for two dimensions, the IGroup algorithm, the R-tree based algorithms, the extensions, as well as the experiments, are all new in this paper.

\section{CONCLUSION}

A new concept, that of concise range queries, has been proposed in this paper, which simultaneously addresses the following three problems of traditional range queries. First, it reduces the query result size significantly as required by the user. The reduced size saves communication bandwidth and also the client's memory and computational resources, which are of the highest importance for mobile devices. Secondly, although the query size has been reduced, the usability of the query results has been actually improved. The concise representation of the results often gives the user more intuitive ideas and enables interactive exploration of the spatial database. Finally, we have designed R-tree based algorithms so that a concise range query can be processed much more efficiently than evaluating the query exactly, especially in terms of I/O cost. This concept, together with its associated techniques presented here, could greatly enhance user experience of spatial databases, especially on mobile devices, by summarizing "the world in a nutshell".

The concept of concise range queries is quite general. One can imagine that it could naturally extend to deal with moving objects [23], [20], [24], uncertainty and fuzziness in data [25], [26], [27], etc. However, designing efficient and effective query processing algorithms for these types of data remains a challenging open problem.

\section{ACKNOWLEDGMENTS}

Ke Yi is supported in part by Hong Kong DAG grant (DAG07/08). Xiang Lian and Lei Chen are supported by Hong Kong RGC Grant No. 611608 and the NSFC Projects under grant No. 60533110 and No. 60763001. Feifei Li is partially supported by the Startup Grant from the Computer Science Department, Florida State University.

\section{References}

[1] X. Lin, Y. Yuan, Q. Zhang, and Y. Zhang, "Selecting stars: The k most representative skyline operator," in ICDE, 2007.

[2] C. Jermaine, S. Arumugam, A. Pol, and A. Dobra, "Scalable approximate query processing with the dbo engine," in SIGMOD, 2007.

[3] G. Ghinita, P. Karras, P. Kalnis, and N. Mamoulis, "Fast data anonymization with low information loss," in VLDB, 2007.

[4] G. Aggarwal, T. Feder, K. Kenthapadi, S. Khuller, R. Panigrahy, D. Thomas, and A. Zhu, "Achieving anonymity via clustering," in PODS, 2006.

[5] J. Xu, W. Wang, J. Pei, X. Wang, B. Shi, and A. W.-C. Fu, “Utilitybased anonymization using local recoding," in SIGKDD, 2006.

[6] C. Böhm, C. Faloutsos, J.-Y. Pan, and C. Plant, "RIC: Parameterfree noise-robust clustering," TKDD, vol. 1, no. 3, 2007.

[7] R. T. Ng and J. Han, "Efficient and effective clustering methods for spatial data mining," in $V L D B, 1994$ 
[8] D. Lichtenstein, "Planar formulae and their uses," SIAM Journal on Computing, vol. 11, no. 2, pp. 329-343, 1982.

[9] R. Tamassia and I. G. Tollis, "Planar grid embedding in linear time," IEEE Transactions on Circuits and Systems, vol. 36, no. 9, pp. $1230-1234,1989$

[10] H. V. Jagadish, B. C. Ooi, K.-L. Tan, C. Yu, and R. Zhang, "iDistance: An adaptive $\mathrm{B}+$-tree based indexing method for nearest neighbor search," ACM Trans. Database Syst., vol. 30, no. 2, pp. 364-397, 2005.

[11] H. Samet, The design and analysis of spatial data structures. Boston, MA, USA: Addison-Wesley Longman Publishing Co., Inc., 1990.

[12] B. Moon, H. v. Jagadish, C. Faloutsos, and J. H. Saltz, "Analysis of the clustering properties of the hilbert space-filling curve," IEEE Trans. on Knowl. and Data Eng., vol. 13, no. 1, pp. 124-141, 2001.

[13] A. Guttman, "R-trees: a dynamic index structure for spatial searching," in SIGMOD, 1984.

[14] N. Beckmann, H. P. Kriegel, R. Schneider, and B. Seeger, "The $\mathrm{R}^{*}$-tree: an efficient and robust access method for points and rectangles," in SIGMOD, 1990.

[15] T. Zhang, R. Ramakrishnan, and M. Livny, "BIRCH: an efficient data clustering method for very large databases," in SIGMOD, 1996.

[16] V. Ganti, R. Ramakrishnan, J. Gehrke, and A. Powell, “Clustering large datasets in arbitrary metric spaces," in ICDE, 1999.

[17] K. Mouratidis, D. Papadias, and S. Papadimitriou, "Tree-based partition querying: a methodology for computing medoids in large spatial datasets," VLDB J., vol. to appear, 2008.

[18] M. Ester, H.-P. Kriegel, J. Sander, and X. Xu, "A density-based algorithm for discovering clusters in large spatial databases with noise," in KDD, 1996.

[19] M. L. Yiu and N. Mamoulis, "Clustering objects on a spatial network," in SIGMOD, 2004.

[20] C. S. Jensen, D. Lin, B. C. Ooi, and R. Zhang, "Effective density queries on continuously moving objects," in ICDE, 2006.

[21] C. R. Palmer and C. Faloutsos, "Density biased sampling: an improved method for data mining and clustering," in SIGMOD, 2000.

[22] K. Yi, X. Lian, F. Li, and L. Chen, "The world in a nutshell: Concise range queries," in ICDE, 2009, short paper.

[23] P. K. Agarwal, L. Arge, and J. Erickson, "Indexing moving points," in PODS, 2000.

[24] Y. Tao, D. Papadias, and J. Sun, "The TPR*-tree: an optimized spatio-temporal access method for predictive queries," in $V L D B$, 2003.

[25] N. Dalvi and D. Suciu, "Efficient query evaluation on probabilistic databases," in VLDB, 2004.

[26] R. Cheng, D. Kalashnikov, and S. Prabhakar, "Evaluating probabilistic queries over imprecise data," in SIGMOD, 2003.

[27] A. D. Sarma, O. Benjelloun, A. Halevy, and J. Widom, "Working models for uncertain data," in ICDE, 2006.

[28] Y. E. Ioannidis and V. Poosala, "Balancing histogram optimality and practicality for query result size estimation," in SIGMOD, 1995.

[29] S. Acharya, V. Poosala, and S. Ramaswamy, "Selectivity estimation in spatial databases," in SIGMOD, 1999.

[30] H. V. Jagadish, N. Koudas, S. Muthukrishnan, V. Poosala, K. C. Sevcik, and T. Suel, "Optimal histograms with quality guarantees," in $V L D B, 1998$.

[31] T. Brinkhoff, "A framework for generating network-based moving objects,", in Geoinformatica, 2002.

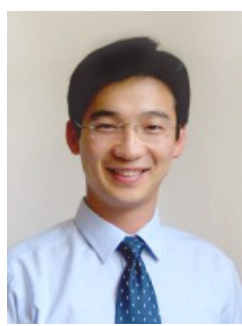

Ke Yi received his B.E. from Tsinghua University and Ph.D. from Duke University, in 2001 and 2006 respectively, both in computer science. After spending one year at AT\&T Labs as a researcher, he has been an Assistant Professor in the Department of Computer Science and Engineering at Hong Kong University of Science and Technology since 2007. His research interests include algorithms, data structures, and databases.

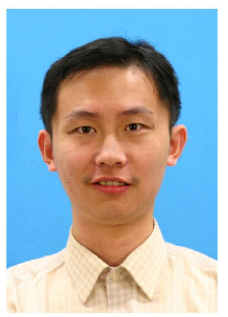

Xiang Lian received the BS degree from the Department of Computer Science and Technology, Nanjing University, in 2003. He is currently a PhD candidate in the Department of Computer Science and Engineering, Hong Kong University of Science and Technology, Hong Kong. His research interests include query processing over stream time series and uncertain databases. $\mathrm{He}$ is a student member of the IEEE.

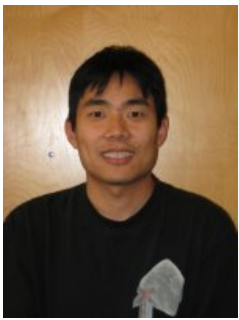

Feifei Li received his B.S. in computer engineering from Nanyang Technological University in 2002 and Ph.D. in computer science from Boston University in 2007 respectively. He has been an Assistant Professor in the Computer Science Department at Florida State University since 2007. His research interests include data management, data structures and databases.

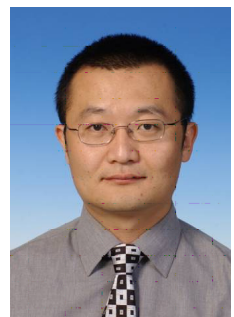

Lei Chen received his BS degree in Computer Science and Engineering from Tianjin University, China, in 1994, the MA degree from Asian Institute of Technology, Thailand, in 1997, and the $\mathrm{PhD}$ degree in computer science from University of Waterloo, Canada, in 2005. He is now an assistant professor in the Department of Computer Science and Engineering at Hong Kong University of Science and Technology. His research interests include uncertain databases, graph databases, multimedia and time series databases, and sensor and peer-to-peer databases. He is a member of the IEEE. 


\section{APPENDIX}

\section{A. Proof of Lemma 2.}

Proof: Let $\mathcal{P}$ be the underlying partitioning of $\mathcal{R}_{\text {opt }}$. Suppose there are $a_{i}$ subsets of size $i$ in $\mathcal{P}, i=1,2, \ldots$ By the properties of $P$ above, we know that the following is a lower bound on $L\left(\mathcal{R}_{\text {opt }}\right)$ :

$f\left(a_{1}, a_{2}, \ldots\right)=0.1 \cdot 2 a_{2}+0.19 \cdot 3 a_{3}+0.24 \cdot 4 a_{4}+0.32 \cdot 5 a_{5}$ $+0.36 \cdot 6 a_{6}+0.38 \cdot\left(7 a_{7}+8 a_{8}+\cdots\right)$.

We wish to minimize $f$ under the following constraints:

$$
\begin{aligned}
a_{1}+a_{2}+a_{3}+\cdots & =k, \\
a_{1}+2 a_{2}+3 a_{3}+\cdots & =|P|=2 k+m .
\end{aligned}
$$

Note that $k>m$. We argue that $f$ achieves minimum when all the $a_{i}$ 's, except $a_{2}$ and $a_{3}$, are zero. Below we perform a case analysis showing that, if any of $a_{1}, a_{4}, a_{5}, \ldots$ is nonzero, then we could further decrease $f$ by reassigning some of the $a_{i}{ }^{\prime}$ s.

- First consider the case $a_{1} \geq 1$. By the constraints (8) and (9), at least one of $a_{3}, a_{4}, \ldots$ must be $\geq 1$. If $a_{3} \geq 1$, we do $a_{1} \leftarrow a_{1}-1, a_{3} \leftarrow a_{3}-1, a_{2} \leftarrow a_{2}+2$, and the change in $f$ is $-0.19 \cdot 3+0.1 \cdot 2 \cdot 2=-0.17$. If $a_{t} \geq 1$ for some $t \geq 4$, we do $a_{1} \leftarrow a_{1}-1, a_{t} \leftarrow a_{t}-1$, $a_{2} \leftarrow a_{2}+1, a_{t-1} \leftarrow a_{t-1}+1$, and the change in $f$ is $\leq-0.24+0.1 \cdot 2=-0.04$.

- Next consider the case $a_{1}=0$. Then $a_{2}$ must be $\geq 1$ due to the constraints (8), (9), and $k>m$. If $a_{4} \geq 1$, we do $a_{2} \leftarrow a_{2}-1, a_{4} \leftarrow a_{4}-1, a_{3} \leftarrow a_{3}+2$, and the change in $f$ is $-0.1 \cdot 2-0.24 \cdot 4+0.19 \cdot 3 \cdot 2=$ -0.02 . If $a_{5} \geq 1$, we do $a_{2} \leftarrow a_{2}-1, a_{5} \leftarrow a_{5}-1$, $a_{3} \leftarrow a_{3}+1, a_{4} \leftarrow a_{4}+1$, and the change in $f$ is $-0.1 \cdot 2-0.32 \cdot 5+0.19 \cdot 3+0.24 \cdot 4=-0.27$. If $a_{6} \geq 1$, we do $a_{2} \leftarrow a_{2}-1, a_{6} \leftarrow a_{6}-1, a_{4} \leftarrow a_{4}+2$, and the change in $f$ is $-0.1 \cdot 2-0.36 \cdot 6+0.24 \cdot 4 \cdot 2=-0.44$. If $a_{t} \geq 1$ for some $t \geq 7$, we first argue that $a_{2} \geq t-3$. By (8) we have $a_{3} \leq k-1-a_{2}$. Since $a_{1}=0$, the LHS of (9) is at least $2 a_{2}+3\left(k-1-a_{2}\right)+t=3 k-3-a_{2}+t$. So we must have

$$
3 k-3-a_{2}+t \leq 2 k+m \leq 3 k,
$$

which gives $a_{2} \geq t-3$. Therefore, we can do $a_{t} \leftarrow$ $a_{t}-1, a_{2} \leftarrow a_{2}-(t-3), a_{3} \leftarrow a_{3}+(t-2)$, which changes $f$ by $-0.38 t-0.1 \cdot 2(t-3)+0.19 \cdot 3(t-2)=$ $-0.01 t-0.54$.

Therefore, $f$ achieves minimum with only $a_{2}$ and $a_{3}$ being nonzero. Then by (8) and (9), $a_{2}$ and $a_{3}$ are uniquely determined as $a_{2}=k-m, a_{3}=m$, which yields $L\left(\mathcal{R}_{\text {opt }}\right) \geq f_{\min }=0.1 \cdot 2 \cdot(k-m)+0.19 \cdot 3 \cdot m=0.2 k+0.37 m$. Furthermore, by Properties 1 and 2, the only way for $\mathcal{R}_{\text {opt }}$ to achieve this minimum is to partition $P$ in the way as stated in the lemma.

\section{B. Proof of Lemma 3.}

Proof: We first prove the "only if" part. Suppose the PLANAR 3-SAT instance has a satisfying assignment, then for each $x_{i}=$ true, we group its chain into $\left\{\left\{p_{i, 1}, p_{i, 2}\right\},\left\{p_{i, 3}, p_{i, 4}\right\}, \ldots,\left\{p_{i, 2 t_{i}-1}, p_{i, 2 t_{i}}\right\}\right\}$, and for each $x_{i}=$ false, we group its chain into $\left\{\left\{p_{i, 2 t_{i}}, p_{i, 1}\right\}\right.$, $\left.\left\{p_{i, 2}, p_{i, 3}\right\}, \ldots,\left\{p_{i, 2 t_{i}-2}, p_{i, 2 t_{i}-1}\right\}\right\}$. This way, for each clause point, the two contacting points in at least one of its joining chains are grouped together. Next we add the clause point into this group. In total, we obtain $m$ groups of 3 with extent 0.19 and $k-m$ groups of 2 with extent 0.1 . This yields a partitioning $\mathcal{R}$ with $L(\mathcal{R})=0.2 k+0.37 m$. By Lemma 2 , this must be optimal.

Next we prove the "if" part. Suppose $L\left(\mathcal{R}_{\text {opt }}\right)=0.2 k+$ $0.37 \mathrm{~m}$. By Lemma 2, this is possible only if each point in any chain is grouped together with one of its adjacent neighbors. Now consider $p_{i, 1}$ and $p_{i, 2}$ for each $i$. If $p_{i, 1}$ and $p_{i, 2}$ are in the same group, then we set $x_{i}=$ true; otherwise we set $x_{i}=$ false. Again by Lemma 2, for each clause point $C_{j}$, the two contacting points in at least one of its joining chains must be grouped together. So $C_{j}$ must be satisfied. 